Pacific

Journal of

Mathematics

\title{
ORDERS OF ELEMENTS IN FINITE QUOTIENTS OF KLEINIAN GROUPS
}

Peter B. Shalen 


\title{
ORDERS OF ELEMENTS IN FINITE QUOTIENTS OF KLEINIAN GROUPS
}

\author{
Peter B. Shalen
}

\begin{abstract}
A positive integer $m$ will be called a finitistic order for an element $\gamma$ of a group $\Gamma$ if there exist a finite group $G$ and a homomorphism $h: \Gamma \rightarrow G$ such that $h(\gamma)$ has order $m$ in $G$. It is shown that up to conjugacy, all but finitely many elements of a given finitely generated, torsion-free Kleinian group admit a given integer $m>2$ as a finitistic order.
\end{abstract}

\section{Introduction}

I will be concerned with the following natural algebraic notion:

Definition 1.1. Let $\gamma$ be an element of a group $\Gamma$. A positive integer $m$ will be called a finitistic order for $\gamma$ if there exist a finite group $G$ and a homomorphism $h: \Gamma \rightarrow G$ such that $h(\gamma)$ has order $m$ in $G$.

1.2. To illustrate this definition, consider the case in which $\Gamma$ is a free abelian group and $\gamma$ is a nontrivial element of $\Gamma$. In this case there is an infinite cyclic direct summand $C$ of $\Gamma$ containing $\gamma$, and hence there is a homomorphism $h_{0}: \Gamma \rightarrow \mathbb{Z}$ such that $k:=h_{0}(\gamma)>0$. Given any positive integer $m$, the quotient homomorphism $\mathbb{Z} \rightarrow \mathbb{Z} / m k \mathbb{Z}$ maps $k$ onto an element of order $m$. Hence any positive integer $m$ is a finitistic order for $\gamma$ in this case.

1.3. On the other hand, if $\gamma$ is an element of finite order $d$ in a group $\Gamma$, it is clear that only divisors of $d$ can be finitistic orders for $\gamma$. In particular, the only finitistic order for the identity element $1 \in \Gamma$ is 1 . Likewise, if a group $\Gamma$ is not residually finite [Magnus 1969], then by definition it contains at least one element $\gamma$ which is in the kernel of every homomorphism from $\Gamma$ to a finite group; the only finitistic order for such an element $\gamma$ is 1 .

By a Kleinian group I will mean a discrete subgroup of $\mathrm{PSL}_{2}(\mathbb{C})$. Such a group may be cocompact; it may be non-cocompact but have finite covolume; or it may have infinite covolume. The main result of this paper says that up to conjugacy,

Partially supported by NSF grant DMS-0906155.

MSC2010: primary 20F99, 57M50; secondary 11R27.

Keywords: 3-manifold, group, finite quotient, order, finitistic order, number field. 
all but finitely many elements of a given finitely generated, torsion-free Kleinian group admit a given integer $m>2$ as a finitistic order. More precisely:

Theorem 1.4. Let $\Gamma$ be a finitely generated, torsion-free Kleinian group. Let $m>2$ be an integer, and let $X$ denote the set of all elements of $\Gamma$ for which $m$ is a finitistic order. Then $\Gamma-X$ is a union of finitely many conjugacy classes.

It is interesting to compare Theorem 1.4 with some of the results proved in [Allman and Hamilton 1999]. It follows from Lemmas 2 and 3 of that reference, together with Proposition 4.2 of the present paper, that if $\Gamma$ is a finitely generated, torsion-free Kleinian group and $m$ is any positive integer, then for every element $\gamma$ of $\Gamma$ there is an integer divisible by $m$ which is a finitistic order for $\gamma$. Note that this result is neither stronger nor weaker than Theorem 1.4.

The most novel ingredient in the proof of Theorem 1.4 is Proposition 2.7 below, which is proved using a deep number-theoretic result, due to Siegel and Mahler, about the finiteness of the set of solutions to the $S$-unit equation in a number field. Proposition 2.7 implies that for an arbitrary integer $m>2$ and an arbitrary finitely generated subgroup $\Gamma$ of $\mathrm{SL}_{2}(E)$, where $E$ is a number field, the traces of those elements of $\Gamma$ that are not of finitistic order $m$ form a finite set. The result is in fact stronger than this because the finite set of exceptional traces does not depend on the group $\Gamma$, but only the smallest number field $K$ containing the traces of all elements of $\Gamma$.

Because Proposition 2.7 does not require discreteness of the group $\Gamma$ and gives finiteness information based only on the "trace field" $K$, it gives information that is not contained in Theorem 1.4. On the other hand, Proposition 2.7 by itself does not directly imply Theorem 1.4, primarily because it establishes finiteness only for the set of exceptional traces and not for the set of exceptional conjugacy classes, and secondarily because it requires $\Gamma$ to be contained in $\operatorname{SL}_{2}(E)$ where $E$ is a number field, rather than in $\mathrm{PSL}_{2}(\mathbb{C})$. Sections 3-5 of this paper are devoted to the geometric arguments that are needed to deduce Theorem 1.4 from the purely algebraic Proposition 2.7. The various ingredients are assembled in Section 6 to prove Theorem 1.4.

Theorem 1.4 is proved by establishing separate finiteness results for the loxodromic elements of a given finitely generated, torsion-free Kleinian group $\Gamma$ which are exceptional (in the sense that they do not admit a given integer $m>2$ as a finitistic order), and for the parabolic elements of $\Gamma$ which are exceptional. The finiteness for the exceptional loxodromic elements is reduced to Proposition 2.7 via results about Kleinian groups established in Section 4. Among these results I would like to call attention to Proposition 4.2, which I have not seen stated before in its general form; it is used to reduce the proof of finiteness for the exceptional loxodromic elements to the special case where the Kleinian group $\Gamma$ is geometrically 
finite, has no rank-1 maximal parabolic subgroups, and is contained in $\operatorname{PSL}_{2}(E)$ for some number field $E$.

The work needed to prove finiteness for exceptional parabolic elements is done in Section 5. The general approach to the main result of this section, Proposition 5.2, was directly inspired by the proof of [Allman and Hamilton 1999, Lemma 3]. The details of the proof turn out to involve Thurston's Dehn filling theorem and some interesting interactions between the topological and geometric aspects of hyperbolic 3-manifolds.

Proposition 2.7 will be applied in a different way to hyperbolic geometry in [Shalen 2011b], which will establish interactions between the Margulis number of a hyperbolic 3-manifold $M$ and the trace field of $M$.

I am grateful to Alan Reid for pointing out to me the result due to Siegel and Mahler which I mentioned above, and to Dick Canary and Steve Kerckhoff for some informative correspondence.

\section{Number fields, traces, and finitistic orders}

2.1. If $K$ is a field and $g$ is an element of $\mathrm{SL}_{2}(K)$, I will denote by $[g]$ the image of $g$ under the quotient homomorphism $\mathrm{SL}_{2}(K) \rightarrow \mathrm{PSL}_{2}(K)$. If $Y$ is a subset of $K$ I will denote by $\mu_{2}(Y)$ the set of all $2 \times 2$ matrices whose entries lie in $Y$. If $Z$ is a subset of $M_{2}(K)$, I will denote by trace $Z$ the set of all traces of elements of $Z$.

Lemma 2.2. Let $m>1$ be an integer, and $k$ be a finite field containing an element $\alpha$ whose order in the multiplicative group $k^{\times}$is $2 m$. Let $g$ be an element of $\mathrm{SL}_{2}(k)$ such that trace $g= \pm\left(\alpha+\alpha^{-1}\right)$. Then $[g] \in \mathrm{PSL}_{2}(k)$ has order $m$.

Proof. First consider the case in which trace $g=\alpha+\alpha^{-1}$. In this case, the characteristic polynomial $X^{2}-\left(\alpha+\alpha^{-1}\right) X+1$ of $g$ has roots $\alpha$ and $\alpha^{-1}$ in $k$, and these roots are distinct since $\alpha$ has order $2 m>1$. Hence $g$ is conjugate in $\mathrm{SL}_{2}(k)$ to $\left(\begin{array}{cc}\alpha & 0 \\ 0 & \alpha^{-1}\end{array}\right)$ and therefore has order $2 m$ in $\mathrm{SL}_{2}(k)$. Since $-I$ is the unique element of order 2 in $\mathrm{SL}_{2}(k)$ it follows that $[g]$ has order $m$ in $\operatorname{PSL}_{2}(k)$.

In the case where trace $g=-\left(\alpha+\alpha^{-1}\right)$, we have trace $(-g)=\alpha+\alpha^{-1}$. By the case already proved it follows that $[g]=[-g]$ has order $m$ in $\operatorname{PSL}_{2}(k)$.

2.3. In this section I will use some concepts and elementary facts from algebraic number theory that will be used. The book [Neukirch 1999] is a general reference.

Let $K$ be a number field. I shall denote by $S_{\infty}(K)$ the set of all archimedean places of $K$.

A set $S$ of places of $K$ will be termed admissible if $S$ is finite and $S \supset S_{\infty}(K)$. If $S$ is admissible, I shall denote by $O_{K, S}$ the ring of $S$-integers of $K$, defined as the intersection of all valuation rings corresponding to places not belonging to $S$. In 
particular, $\mathscr{O}_{K}:=\mathscr{O}_{K, \varnothing}$ is the ring of integers of $K$ (see the statement $(\alpha)$ on p. 264 of [Ribenboim 1999]).

2.4. If $K$ and $E$ are number fields, with $K \subset E$, and if $S$ is an admissible set of places in a number field $K$, I shall denote by $S^{E}$ the set of all extensions to $E$ of places in $S$. Since a given valuation of $K$ admits only finitely many extensions to valuations of $E$, for example by Proposition 8.2 of [Neukirch 1999], $S^{E}$ is an admissible set of places of $E$. On the other hand, since (by the same proposition) every valuation of $K$ admits at least one extension to a valuation of $E$, we have $0_{K, S}=0_{E, S} \cap K$.

2.5. Let $E$ be a number field, and let $\mathfrak{p}$ be a nonarchimedean place of $E$. I will denote by $\mathfrak{o}_{(\mathfrak{p})}$ the valuation ring defined by $\mathfrak{p}$, by $k_{\mathfrak{p}}$ the residue field $\mathfrak{o}_{(\mathfrak{p})} / \mathfrak{p}$, and by $\eta_{\mathfrak{p}}: \mathfrak{o}_{(\mathfrak{p})} \rightarrow k_{\mathfrak{p}}$ the quotient homomorphism. I will denote by $h_{\mathfrak{p}}$ the natural homomorphism $\mathrm{SL}_{2}\left(\mathfrak{o}_{(\mathfrak{p})}\right) \rightarrow \mathrm{SL}_{2}\left(k_{\mathfrak{p}}\right)$, defined by

$$
\left(\begin{array}{ll}
a & b \\
c & d
\end{array}\right) \mapsto\left(\begin{array}{ll}
\eta_{\mathfrak{p}}(a) & \eta_{\mathfrak{p}}(b) \\
\eta_{\mathfrak{p}}(c) & \eta_{\mathfrak{p}}(d)
\end{array}\right)
$$

I will denote by $\kappa_{\mathfrak{p}}: \mathrm{SL}_{2}\left(\mathfrak{o}_{(\mathfrak{p})}\right) \rightarrow \operatorname{PSL}_{2}\left(k_{\mathfrak{p}}\right)$ the homomorphism defined by $\kappa_{\mathfrak{p}}(\gamma)=$ $\left[h_{\mathfrak{p}}(\gamma)\right]$.

Lemma 2.6. For each integer $n>1$ there is a positive integer $N=N(n)$ with the following property. Let $E$ be any number field, and let $\mathfrak{p}$ be a nonarchimededean place of $E$ such that $1 / N \in \mathfrak{o}_{(\mathfrak{p})}$. Let $\omega \in E$ be a root of unity of order $n$. Then $\omega \in O_{E} \subset \mathfrak{o}_{(\mathfrak{p})}$, and $\eta_{\mathfrak{p}}(\omega)$ is an element of order $n$ in the multiplicative group $k_{\mathfrak{p}}^{\times}$.

Proof. Let $n$ be a positive integer. The roots of the cyclotomic polynomial $\Phi_{n}(X) \in$ $\mathbb{Z}[X]$ are the primitive $n$-th roots of unity. For each proper divisor $d$ of $n$, the roots of $X^{d}-1$ are the $d$-th roots of unity. Hence $X^{d}-1$ is relatively prime to $\Phi_{n}(X)$ in $\mathbb{Q}[X]$, and so there exist polynomials $A_{d}(X)$ and $B_{d}(X)$ in $\mathbb{Z}[X]$ such that $A_{d}(X) \Phi_{n}(X)+B_{d}(X)\left(X^{d}-1\right)=N_{d}$ for some nonzero integer $N_{d}$. I will take $N$ to be a positive integer which is divisible by $N_{d}$ for every proper divisor $d$ of $n$, and show that the conclusion holds with this choice of $N$.

Let $E$ be any number field, and let $\mathfrak{p}$ be a nonarchimededean place of $E$ such that $1 / N \in \mathfrak{o}_{(\mathfrak{p})}$. Let $\omega \in E$ be a root of unity of order $n$. Then in particular $\omega$ is an algebraic integer, and so $\omega \in \mathfrak{O}_{E} \subset \mathfrak{o}_{(\mathfrak{p})}$. Let us set $\alpha=\eta_{\mathfrak{p}}(\omega)$. Since $\eta_{\mathfrak{p}}: \mathfrak{o}_{(\mathfrak{p})} \rightarrow k_{\mathfrak{p}}$ is homomorphism, we have $\alpha^{n}=1$. I shall complete the proof by assuming that the order of $\alpha$ is a proper divisor $d$ of $n$ and deriving a contradiction.

Since the equality $A_{d}(X) \Phi_{n}(X)+B_{d}(X)\left(X^{d}-1\right)=N_{d}$ holds in $\mathbb{Z}[X]$, and since $N_{d} \mid N$ and $1 / N \in \mathfrak{o}_{(\mathfrak{p})}$, we have $A_{d}(\alpha) \Phi_{n}(\alpha)+B_{d}(\alpha)\left(\alpha^{d}-1\right) \neq 0$ in $k_{\mathfrak{p}}$. But since $\omega$ has order $n$ in $E^{\times}$, we have $\Phi_{n}(\omega)=0$, and since $\eta_{\mathfrak{p}}$ is a homomorphism we have $\Phi_{n}(\alpha)=0$. Furthermore, since $\alpha$ has order $d$ in $k_{\mathfrak{p}}^{\times}$, we have $\alpha^{d}-1=0$. This is a contradiction. 
I will now turn to the main result of Section 2.

Proposition 2.7. Let $K$ be a number field, let $S$ be an admissible set of places of $K$, and let $m>2$ be an integer. Then there is a finite set $W \subset \mathrm{O}_{K, S}$ with the following property. Let $\gamma$ be an element of $\mathrm{SL}_{2}\left(O_{K, S}\right)$ such that trace $\gamma \notin W$. Then there exists a place $\mathfrak{p}$ of $K$, with $\mathfrak{p} \notin S$, such that $\kappa_{\mathfrak{p}}(\gamma) \in \mathrm{PSL}_{2}\left(k_{\mathfrak{p}}\right)$ has order $m$. In particular, $m$ is a finitistic order for $\gamma \in \mathrm{SL}_{2}\left(\mathrm{O}_{K, S}\right)$.

Proof. Set $n=2 m$. Let $N=N(n)$ be a positive integer having the property stated in Lemma 2.6. Let $K_{0}$ be a finite extension of $K$ which contains a primitive $n$-th root of unity $\omega$, and set $\tau=\omega+\omega^{-1} \in K_{0}$. Since $m>2$ we have $\omega^{2} \neq-1$ and hence $\tau \neq 0$. Let $S_{0}$ denote an admissible set of places of $K_{0}$, containing $S^{K_{0}}$ (see Section 2.4), such that $2, N$ and $\tau$ are units in $\mathcal{O}_{K_{0}, S_{0}}$.

Let $U$ denote the set of all elements $u \in 0_{K_{0}, S_{0}}$ such that both $u$ and $1-u$ are units in $O_{K_{0}, S_{0}}$. According to [Hindry and Silverman 2000, Theorem D.8.1] (a result due to Siegel and Mahler), $U$ is a finite set. Hence the set $W_{0}:=\{(2 u-1) \tau: u \in U\} \subset K_{0}$ is also finite, and so is $W=K \cap W_{0}$. I shall complete the proof of the proposition by showing that the conclusion is true with this choice of $W$.

I claim that for any $t \in K_{0}-W_{0}$, either $t-\tau$ or $t+\tau$ is a nonunit in $\mathrm{O}_{K_{0}}, S_{0}$. Indeed, suppose that $t-\tau$ and $t+\tau$ are both units. Since 2 and $\tau$ are also units in $\mathcal{O}_{K_{0}, S_{0}}$, it then follows that $u:=(\tau+t) / 2 \tau$ and $1-u=(\tau-t) / 2 \tau$ are units as well, i.e., that $u \in U$. Hence $t=(2 u-1) \tau \in W_{0}$, a contradiction.

Now let $\gamma$ be any element of $\mathrm{SL}_{2}\left(\mathrm{O}_{K, S}\right)$ such that $t:=\operatorname{trace} \gamma \notin W$. In particular we have $t \in K_{0}-W_{0}$. Hence we may choose an element $\epsilon$ of $\{1,-1\}$ such that $t-\epsilon \tau$ is a nonunit in $\mathrm{O}_{K_{0}}, S_{0}$. It follows that there is a place $\mathfrak{p}_{0}$ of $K_{0}$, with $\mathfrak{p}_{0} \notin S_{0}$, such that $t-\epsilon \tau$ belongs to the maximal ideal $\mathfrak{m}_{0}$ of $\mathfrak{o}_{\left(\mathfrak{p}_{0}\right)}$. Since $\mathfrak{p}_{0} \notin S_{0}$, we have $\mathfrak{O}_{K_{0}, S_{0}} \subset \mathfrak{o}_{\left(\mathfrak{p}_{0}\right)}$. Since $t-\epsilon \tau \in \mathfrak{m}_{0}$, we have, in the notation of Section 2.5, $\eta_{\mathfrak{p}_{0}}(t)=\epsilon \eta_{\mathfrak{p}_{0}}(\tau) \in k_{\mathfrak{p}_{0}}$. But since $t=\operatorname{trace} \gamma$ we have $\eta_{\mathfrak{p}_{0}}(t)=$ trace $h_{\mathfrak{p}_{0}}(\gamma)$. Thus the element $g:=h_{\mathfrak{p}_{0}}(\gamma)$ of $\operatorname{SL}_{2}\left(k_{\mathfrak{p}_{0}}\right)$ has trace $\epsilon \eta_{\mathfrak{p}_{0}}(\tau)$. If we set $\alpha=\eta_{\mathfrak{p}_{0}}(\omega)$, then $\eta_{\mathfrak{p}_{0}}(\tau)=\alpha+\alpha^{-1}$. Since $\omega$ is a primitive $n$-th root of unity, and since $1 / N \in \mathbb{O}_{K_{0}, S_{0}} \subset \mathfrak{o}_{\left(\mathfrak{p}_{0}\right)}$, it follows from Lemma 2.6 that $\alpha$ has order $n=2 m$ in $k_{\mathfrak{p}_{0}}^{\times}$. Since trace $g=\epsilon\left(\alpha+\alpha^{-1}\right)$, it follows from Lemma 2.2 that $[g]=\kappa_{\mathfrak{p}_{0}}(\gamma) \in \operatorname{PSL}_{2}\left(k_{\mathfrak{p}_{0}}\right)$ has order $m$.

We have ker $\kappa_{\mathfrak{p}_{0}}=\{ \pm I\}+\mathcal{M}_{2}\left(\mathfrak{m}_{0}\right)$. Let $\mathfrak{p}$ denote the restriction of the place $\mathfrak{p}_{0}$ to $K$. Since $\mathfrak{p}_{0} \notin S_{0}$, and since $S^{K_{0}} \subset S$, we have $\mathfrak{p} \notin S$. If $\mathfrak{m}$ denotes the maximal ideal of $\mathfrak{o}_{(\mathfrak{p})}$, we have ker $\kappa_{\mathfrak{p}}=\{ \pm I\}+\mathcal{M}_{2}(\mathfrak{m})$. Hence $\operatorname{ker} \kappa_{\mathfrak{p}}=\mathrm{SL}_{2}\left(\mathfrak{o}_{(\mathfrak{p})}\right) \cap \operatorname{ker} \kappa_{\mathfrak{p}_{0}}$. It follows that $\kappa_{\mathfrak{p}}(\gamma)$ has the same order as $\kappa_{\mathfrak{p}_{0}}(\gamma)$, namely $m$.

\section{Preliminaries on Kleinian groups}

The study of torsion-free Kleinian groups is closely related to the study of (complete) orientable hyperbolic 3-manifolds. The topological group $\mathrm{PSL}_{2}(\mathbb{C})$ may be identified by a continuous isomorphism with the group of orientation-preserving 
isometries of the 3-dimensional hyperbolic space $\mathbb{H}^{3}$. If $\Gamma \leq \mathrm{PSL}_{2}(\mathbb{C})$ is discrete and torsion-free, the action of $\Gamma$ on $\mathbb{H}^{3}$ is free and properly discontinuous, and $M:=\mathbb{T}^{3} / \Gamma$ inherits the structure of an orientable hyperbolic 3-manifold with $\pi_{1}(M) \cong \Gamma$. Up to conjugacy there is a natural identification of $\pi_{1}(M)$ with $\Gamma$.

In this section I will collect a few essential facts about hyperbolic 3-manifolds which will be needed in Sections 4 and 5.

3.1. I will follow the conventions of [Shalen 2010, Section 3]. In particular, I will work in the smooth category (so that manifolds and submanifolds are understood to be smooth) but will often quote results proved in the piecewise linear category; the justification for doing this is explained in Section 3.1 of [Shalen 2010]. It is understood that a manifold may have a boundary. Recall that a 3-manifold $M$ is said to be irreducible if $M$ is connected and every 2 -sphere in $M$ is the boundary of a 3-ball in $M$. According to [Shalen 2010, Proposition 3.8], every orientable hyperbolic 3-manifold is irreducible.

I will also follow the conventions of [Shalen 2010, Section 2] in statements and arguments involving fundamental groups: I will suppress base points whenever it is possible to do so without ambiguity.

An element of $\mathrm{PSL}_{2}(\mathbb{C})$ is said to be parabolic if it is nontrivial and has the form $[A]$ (see Section 2.1) for some $A \in \mathrm{SL}_{2}(\mathbb{C})$ with trace $A=2$. By a parabolic subgroup of a Kleinian group $\Gamma$ I will mean a nontrivial subgroup of $\Gamma$ whose nontrivial elements are all parabolic.

By a standard cusp neighborhood $X$ in the orientable hyperbolic 3-manifold $M=\mathbb{M}^{3} / \Gamma$ I will mean a subset of the form $B / \Gamma_{B}$, where $B$ is an open horoball in $\mathbb{H}^{3}$, precisely invariant under $\Gamma$ (in the sense that $\gamma \cdot B$ is either equal to $B$ or disjoint from $B$ for every $\gamma \in \Gamma$ ), and the stabilizer $\Gamma_{B}$ of $B$ is a parabolic subgroup. I will define the rank of $X$ to be the rank of $\Gamma_{B}$ (which must be equal to 1 or 2).

In general a Kleinian group is said to be elementary if it has an abelian subgroup of finite index. A torsion-free elementary Kleinian group is itself abelian (see, e.g., [Shalen 2011a, Proposition 2.1]).

3.2. A nonelementary, orientable hyperbolic 3-manifold $M$ has a well-defined convex core, which I will denote by $\mathscr{C}(M)$. By definition, $\mathscr{C}(M)$ is the smallest nonempty closed subset of $M$ which is convex in the strong sense that every geodesic path with endpoints in $\mathscr{C}(M)$ is entirely contained in $\mathscr{C}(M)$. For a construction of $\mathscr{C}(M)$ and proofs of its basic properties, see [Matsuzaki and Taniguchi 1998, Subsection 3.1.1] or [Morgan 1984, p. 63]. I will denote by $\mathscr{C}_{1}(M)$ the closed radius-1 metric neighborhood of $\mathscr{C}_{1}(M)$; it follows from [Matsuzaki and Taniguchi 1998, Proposition 3.1] that $\mathscr{C}_{1}(M)$ is a 3-manifold and a deformation retract of $M$.

A closed geodesic in a hyperbolic manifold $M$ will be regarded as a map $C$ : $S^{1} \rightarrow M$ such that the map $t \mapsto C\left(e^{2 \pi i t}\right)$ from $\mathbb{R}$ to $M$ is a geodesic; I will write 
$|C|=C\left(S^{1}\right)$. The construction of $\mathscr{C}(M)$ given by Matsuzaki and Taniguchi or by Morgan immediately implies that $|C| \subset \mathscr{C}(M)$ for any closed geodesic $C$.

3.3. As in [Matsuzaki and Taniguchi 1998, p. 55], I will define the $\epsilon$-thin part $M_{(0, \epsilon)}$ of a nonelementary orientable hyperbolic 3-manifold $M$, where $\epsilon>0$ is given, to be the set of all points of $M$ which are base points of homotopically nontrivial loops of length less than $\epsilon$. According to Theorem 2.24 of the same work, there is a universal constant $\epsilon_{0}$ such that for every orientable 3-manifold $M$, each component of $M_{\left(0, \epsilon_{0}\right)}$ is either a standard cusp neighborhood or a metric neighborhood of a simple closed geodesic. I will fix such a constant $\epsilon_{0}$ for the rest of the paper. According to [Morgan 1984, Lemma 6.7], each rank-2 standard cusp neighborhood $X$ in $M$ contains a smaller rank-2 standard cusp neighborhood $s(X)$ which is contained in $\mathscr{C}(M)$. I will refer to the set $K:=\mathscr{C}_{1}(M)-\bigcup_{X} s(X)$, where $X$ ranges over all components of $M_{\left(0, \epsilon_{0}\right)}$ which are rank-2 standard cusp neighborhoods, as a truncation of $\mathscr{C}_{1}(M)$. Thus every nonelementary orientable hyperbolic 3-manifold admits a truncation $K$. (According to this definition, a truncation of $\mathscr{C}_{1}(M)$ is not quite unique, as it depends on the choice of the standard cusp neighborhoods $s(X)$.)

3.4. Let $K$ be an orientable, irreducible 3-manifold. An essential singular torus in $K$ is defined to be a map $f: T^{2} \rightarrow K$ such that (i) $f_{\sharp}: \pi_{1}\left(T^{2}\right) \rightarrow \pi_{1}(K)$ is injective, and (ii) $f$ is not homotopic in $K$ to a map of $T^{2}$ into $\partial K$.

Now suppose that $\mathscr{T}$ is a 2 -dimensional submanifold of $\partial K$ such that the inclusion homomorphism $\pi_{1}(T) \rightarrow \pi_{1}(K)$ is injective for every component $T$ of $\mathscr{T}$. In this setting I will define an essential singular annulus in the pair $(K, \mathscr{T})$ to be a map of pairs $f:\left(S^{1} \times[0,1], \partial\left(S^{1} \times[0,1]\right)\right) \rightarrow(K, \mathscr{T})$ such that $f_{\sharp}: \pi_{1}\left(S^{1} \times[0,1]\right) \rightarrow$ $\pi_{1}(K)$ is injective, and $f$ is not homotopic rel $\partial\left(S^{1} \times[0,1]\right)$ to a map of $\left.S^{1} \times[0,1]\right)$ into $\mathscr{T}$.)

Proposition 3.5. Let $M=\mathbb{M}^{3} / \Gamma$ be an orientable hyperbolic 3-manifold, and let $K$ be a truncation of $\mathscr{C}_{1}(M)$. Then:

(1) $K$ is irreducible and has no essential singular tori, and is a deformation retract of $M$.

(2) For every torus component $T$ of $\partial K$ there is a standard cusp neighborhood which is a component of $\overline{M-K}$ and is bounded by $T$; furthermore, the inclusion homomorphism $\pi_{1}(T) \rightarrow \pi_{1}(M)$ is injective, and its image, a subgroup of $\pi_{1}(M)=\Gamma$ defined up to conjugacy, is a rank-2 maximal parabolic subgroup of $\Gamma$.

(3) Conversely, every rank-2 maximal parabolic subgroup of $\Gamma$ is conjugate to the image of the inclusion homomorphism $\pi_{1}(T) \rightarrow \pi_{1}(M)$ for some torus component $T$ of $\partial K$. 
Proof. Let $p: \mathbb{M}^{3} \rightarrow M$ denote the quotient projection.

Suppose that $P$ is a rank-2 maximal parabolic subgroup of $\Gamma$. Fix generators $\gamma_{1}$ and $\gamma_{2}$ of $P$. Since $\gamma_{1}$ and $\gamma_{2}$ are commuting parabolics, there is a point $\tilde{x} \in \mathbb{M}^{3}$ such that $d\left(\tilde{x}, \gamma_{i} \cdot \tilde{x}\right)<\epsilon_{0}$ for $i=1,2$. Set $x=p(\tilde{x})$. The base point $\tilde{x} \in p^{-1}(x) \subset$ $\mathbb{M}^{3}$ determines an isomorphic identification of $\pi_{1}(M, x)$ with $\Gamma$, and under this identification, each $\gamma_{i}$ is represented by a loop $c_{i}:[0,1] \rightarrow M$ of length $<\epsilon_{0}$. If we set $G=c_{1}([0,1]) \cup c_{2}([0,1])$, then each point of $G$ is the basepoint of a loop of length $<\epsilon_{0}$, and hence $G \subset M_{\left(0, \epsilon_{0}\right)}$. Under our identification, $P$ is contained in the image $P^{\prime}$ of the inclusion homomorphism $\pi_{1}(G, x) \rightarrow \pi_{1}(M, x)$, and hence in the image of the inclusion homomorphism $\pi_{1}(X, x) \rightarrow \pi_{1}(M, x)$, where $X$ denotes the component of $M_{\left(0, \epsilon_{0}\right)}$ containing $G$. Since $P$ is non-cyclic, the discussion in Section 3.3 shows that $X$ is a rank-2 standard cusp neighborhood; hence $P^{\prime}$ is a parabolic subgroup of $\Gamma$, and the maximality of $P$ implies that $P=P^{\prime}$. It now follows from the definition of a truncation that $X$ contains a unique torus component $T$ of $\partial K$, and that the image (a priori defined up to conjugacy) of the inclusion homomorphism $\pi_{1}(T) \rightarrow \pi_{1}(K)$ is conjugate to $P$. This proves (3).

Conversely, suppose that $T$ is a torus component of $\partial K$. According to the definition of a truncation, $T$ is either (a) a component of $\partial \mathscr{C}_{1}(M)$, or (b) the boundary of a standard cusp neighborhood which is a component of $\overline{M-K}$. However, by [Matsuzaki and Taniguchi 1998, Proposition 3.1], $\partial \mathscr{C}_{1}(M)$ is homeomorphic to $\Omega / \Gamma$, where $\Omega$ denotes the set of discontinuity of $\Gamma$. Hence if (a) holds, some component of $\Omega$ conformally covers a torus; this is impossible for a nonelementary Kleinian group $\Gamma$, because the limit set $\Lambda=\widehat{\mathbb{C}}-\Omega$ must contain more than two points, and hence each component of $\Gamma$ is a hyperbolic Riemann surface. Hence (b) must hold. If $X$ denotes the standard cusp neighborhood bounded by $T$, then by definition $p^{-1}(X)$ is a horoball $B \subset \mathbb{M}^{3}$, and $p^{-1}(T)$ is the frontier $H$ of $B$ in $\mathbb{T}^{3}$. Since $H$ is simply connected, the inclusion homomorphism $i: \pi_{1}(T) \rightarrow \pi_{1}(M)$ is injective. Furthermore, the image of $i$ is identified with the stabilizer $\Gamma_{B}$ of $B$ in $\Gamma$, which is a maximal parabolic subgroup of $\Gamma=\pi_{1}(M)$; being isomorphic to $\pi_{1}(T)$, it has rank 2 . This proves (2).

To prove (1), first note that according to the definition of a truncation, each component of $\mathscr{C}_{1}(M)-K$ is a standard cusp neighborhood bounded by a torus component of $\partial K$; hence $K$ is a deformation retract of $\mathscr{C}_{1}(M)$. Since $\mathscr{C}_{1}(M)$ is in turn a deformation retract of $M$ (cf. Section 3.2), it follows that $K$ is a deformation retract of $M$.

Next note that, by [Matsuzaki and Taniguchi 1998, Proposition 3.1], $\mathscr{C}_{1}(M)$ is a deformation retract of $M$; hence every component of $M-\mathscr{C}_{1}(M)$ has noncompact closure in $M$. In view of the definition of a truncation, it follows that every component of $M-K$ has noncompact closure in $M$. Since $M$ is irreducible by [Shalen 2010, Proposition 3.8], it follows that $K$ is irreducible. Finally, suppose 
that $f: T^{2} \rightarrow K \subset M$ is an essential singular torus. Then $X:=f_{\sharp}\left(\pi_{1}\left(T^{2}\right)\right) \leq \pi_{1}(M)$ is a rank-2 free abelian subgroup of $\pi_{1}(M)$ which is defined up to conjugacy. There is an isomorphic identification of $\pi_{1}(M)$ with $\Gamma$ which is also canonically defined up to conjugacy. Since $\Gamma$ is discrete, $X$ must be parabolic. Let $X_{0}$ be a maximal parabolic subgroup containing $X$; as a parabolic subgroup, $X_{0}$ is free abelian of rank at most 2, and since it contains $X$ its rank must be exactly 2. By assertion (3) of the proposition, which was proved above, $X_{0}$ is conjugate to the image of the inclusion homomorphism $\pi_{1}(T) \rightarrow \pi_{1}(M)$ for some torus component $T$ of $\partial K$. In particular, $X$ is conjugate to a subgroup of the image of the inclusion homomorphism $\pi_{1}(T) \rightarrow \pi_{1}(M)$. Since $K$ is a deformation retract of $M$ by Proposition 3.5 and is therefore aspherical, it follows that $f$ is homotopic, in $K$, to a map of $T^{2}$ into $T$. This contradicts the definition of an essential singular torus.

3.6. An orientable hyperbolic 3-manifold $M=\mathbb{H}^{3} / \Gamma$ (or the Kleinian group $\Gamma$ ) is said to be geometrically finite if $M$ is nonelementary and $\mathscr{C}_{1}(M)$ has a compact truncation.

Proposition 3.7. An orientable hyperbolic 3-manifold $M$ has finite volume if and only if $M$ is geometrically finite and $\mathscr{C}_{1}(M)$ has a truncation whose boundary components are all tori.

Proof. If $\mathscr{C}_{1}(M)$ has a truncation whose boundary components are all tori, it follows from Assertion (2) of Proposition 3.5 that $\mathscr{C}_{1}(M)$ has no boundary; hence $\mathscr{C}_{1}(M)=$ $M$. If in addition $M$ is geometrically finite, then $\mathscr{C}_{1}(M)$ has finite volume according to Proposition 3.7 of [Matsuzaki and Taniguchi 1998]; hence in this case $M$ has finite volume. Conversely, if $M$ has finite volume, then in particular $\mathscr{C}_{1}(M)$ has finite volume, and the proposition just cited implies that $M$ is geometrically finite. Furthermore, if we write $M=\mathbb{M}^{3} / \Gamma$, the finiteness of vol $M$ implies that the limit set of $\Gamma$ is the entire sphere at infinity; hence by [Matsuzaki and Taniguchi 1998, Proposition 3.1], $\mathscr{C}_{1}(M)$ has no boundary, and so the boundary components of a truncation of $\mathscr{C}_{1}(M)$ are all tori.

An orientable hyperbolic 3-manifold $M=\mathbb{M}^{3} / \Gamma$ will be said to have no rank-1 cusps if every maximal parabolic subgroup of $\Gamma$ has rank 2 .

The following version of Thurston's geometrization theorem is a kind of converse to Proposition 3.5.

Proposition 3.8. Let $K$ be a compact, irreducible orientable 3-manifold which has nonempty boundary and has no essential singular tori. Then either $\pi_{1}(K)$ is isomorphic to either a Klein bottle group $\left\langle x, y: y x y^{-1}=x^{-1}\right\rangle$ or a free abelian group of rank at most 2 , or $K$ is diffeomorphic to a truncation of $\mathfrak{b}_{1}(M)$ for some geometrically finite orientable hyperbolic 3-manifold $M$ having no rank-1 cusps. 
Proof. Let $\mathscr{T}$ denote the union of all torus components of $K$. If $\mathscr{T}$ has a component $T$ such that the inclusion homomorphism $\pi_{1}(T) \rightarrow \pi_{1}(K)$ is not injective, then $\pi_{1}(K)$ is infinite cyclic by [Shalen 2010, Proposition 3.10]. We may therefore assume that $\pi_{1}(T) \rightarrow \pi_{1}(K)$ is injective for every component $T$ of $\mathscr{T}$. We may also assume that $K$ is not a 3-ball, as otherwise $\pi_{1}(K)$ is trivial.

Consider the case in which the pair $(K, \mathscr{T})$ has no essential singular annuli Section 3.4. In this case, since $K$ also has no essential singular tori, and since $\pi_{1}(T) \rightarrow \pi_{1}(K)$ is injective for every component $T$ of $\mathscr{T}$, the pair $(K, \mathscr{T})$ is a pared manifold in the sense of [Morgan 1984, Definition 4.8]. Furthermore, since $K$ is irreducible and is not a ball, and $\partial K \neq \varnothing$, the manifold $K$ is a Haken manifold in the sense defined on page 57 of [Morgan 1984]. Hence the pared manifold $(K, \mathscr{T})$ satisfies the hypotheses of Theorem $\mathrm{B}^{\prime}$ in [Morgan 1984, p. 70]. Since the components of $\mathcal{T}$ (if any) are all tori (rather than annuli), the conclusion of that theorem may be expressed, in the language of the present paper, by saying that there is a geometrically finite orientable hyperbolic manifold $M$, having no rank-1 cusps, such that $K$ is diffeomorphic to a truncation of $\mathscr{C}_{1}(M)$.

There remains the case in which the pair $(K, \mathscr{T})$ has an essential singular annulus $f:\left(S^{1} \times[0,1], \partial\left(S^{1} \times[0,1]\right)\right) \rightarrow(K, \mathscr{T})$. I will use the terminology of [Jaco and Shalen 1979] in the following argument. Let $(\Sigma, \Phi)$ denote a characteristic pair of the compact, irreducible pair $(K, \mathscr{T})$, which exists by [ibid., p. 138]. According to [ibid., Remark IV.1.2] and the definition of a characteristic pair ([ibid., p. 138]), after modifying $f$ by a homotopy of maps of pairs, we may assume that $f\left(S^{1} \times[0,1]\right) \subset \Sigma$ and $f\left(\partial\left(S^{1} \times[0,1]\right)\right) \subset \Phi$. Let $\Sigma_{0}$ denote the component of $\Sigma$ containing $f\left(S^{1} \times[0,1]\right)$.

According to the definition of a characteristic pair, $\Sigma_{0}$ is a "perfectly embedded pair" in the sense of [ibid., p. 4]. Thus each component of $\Phi_{0}:=\Sigma_{0} \cap \partial M=\Sigma_{0} \cap \mathscr{T}$ is a compact submanifold of $\mathscr{T}$ whose boundary curves (if any) are homotopically nontrivial in $\mathscr{T}$. Since the components of $\mathscr{T}$ are tori, each component of $\Phi_{0}$ is a torus or an annulus.

According to the definition of a characteristic pair, either $\Sigma_{0}$ is a Seifert fibered space and $\Phi_{0} \subset \Sigma_{0}$ is saturated, or $\Sigma_{0}$ is a [0,1]-bundle whose associated $\{0,1\}$ bundle is $\Phi_{0}$. In the latter subcase, since the components of $\Phi_{0}$ are tori and annulus, the base of the [0,1]-bundle is a torus, Klein bottle, annulus or Möbius band, and hence the $[0,1]$-bundle may be given the structure of a Seifert fibered space in such a way that $\Phi_{0}$ is saturated. Thus in any event $\Sigma_{0}$ is a Seifert fibered space and $\Phi_{0}$ is saturated.

I claim that the components of $\Phi_{0}$ are tori. To prove this, let $\Sigma_{1}$ denote a regular neighborhood of the union of $\Sigma_{0}$ with all those components of $\mathscr{T}$ which meet $\Sigma_{0}$. Since $\Phi_{0}$ is saturated in $\Sigma_{0}$, the manifold $\Sigma_{1}$ is a Seifert fibered space. Since $\Sigma_{1} \cap \mathscr{T}$ is a union of boundary tori of $\Sigma_{1}$, the pair $\left(\Sigma_{1}, \Sigma_{1} \cap \mathscr{T}\right)$ is a Seifert pair. 
Since $f$ is an essential singular annulus and $f\left(S^{1} \times[0,1]\right) \subset \Sigma_{0}$, the inclusion map $i:\left(\Sigma_{1}, \Sigma_{1} \cap \mathscr{T}\right) \rightarrow(K, \mathscr{T})$ is an essential, nondegenerate map of Seifert pairs. The defining property of the characteristic pair therefore implies that $i$ is homotopic as a map of pairs to a map $i^{\prime}$ such that $i^{\prime}\left(\Sigma_{1}\right) \subset \Sigma_{0}$ and $i^{\prime}\left(\Sigma_{1} \cap \mathscr{T}\right) \subset \Phi_{0}$. In particular, the inclusion of $\Sigma_{1} \cap \mathcal{T}$ into $\mathscr{T}$ is homotopic in $\mathscr{T}$ to a map whose image is contained in $\Phi_{0}$. Since the components of $\Sigma_{1} \cap \mathcal{T}$ are closed surfaces, it follows that $\Sigma_{1} \cap \mathcal{T}=\Phi_{0}$, i.e., that the components of $\Phi_{0}$ are tori.

Now I claim that $K=\Sigma_{0}$. If this is false, we may fix a component $C$ of the frontier of $\Sigma_{0}$ in $K$. Since $\Sigma_{0}$ is a Seifert fibered space and $\Sigma \cap \partial K=\Phi_{0}$ is a union of components of $\partial \Sigma_{0}$, the surface $C$ is a torus. Since $\Sigma_{0}$ is perfectly embedded, the inclusion homomorphism $\pi_{1}(C) \rightarrow \pi_{1}(M)$ is injective. As $K$ contains no essential tori, the inclusion $C \rightarrow K$ must be homotopic to a map of $C$ into $\partial K$. It then follows from [Waldhausen 1968, Corollary 5.5] that $C$ is boundary-parallel in $K$. This contradicts the definition of a perfectly embedded pair.

Since $K=\Sigma_{0}$, the manifold $K$ is a Seifert fibered space. Hence $\Gamma:=\pi_{1}(K)$ has a cyclic normal subgroup $N$ such that $Q:=\Gamma / N$ is a Fuchsian group. Let $\widetilde{\Gamma}$ denote the centralizer of $N$ in $\Gamma$, so that $[\Gamma: \widetilde{\Gamma}] \leq 2$. Let $q: \Gamma \rightarrow Q$ denote the quotient projection, and set $\widetilde{Q}=q(\widetilde{\Gamma})$. Since $\partial K \neq \varnothing$, we may write $Q$ as a free product of nontrivial cyclic groups.

If in the free product description of $Q$ there are at least three factors, or if there are at least two factors and one of them order strictly greater than 2 , then $\widetilde{Q}$ has infinitely many nonconjugate maximal infinite cyclic subgroups. For each maximal cyclic subgroup $Z$ of $\widetilde{Q}$, the group $q^{-1}(Q)$ is a maximal rank-2 free abelian subgroup of $\Gamma$. Hence $\Gamma$ has infinitely many nonconjugate maximal rank-2 free abelian subgroups. As $\partial K$ has only finitely many components, it follows that $K$ admits an essential singular torus, a contradiction to the hypothesis.

Hence in the free product description of $Q$ there are at most two factors, and if there are two factors they are both of order 2 . This means that either the base $B$ of the Seifert fibration is a Möbius band or annulus and there are no singular fibers; or $B$ is a disk and there is at most one singular fiber; or $B$ is a disk and there are two singular fibers, both of local degree 2. It follows that $K$ is diffeomorphic to a solid torus - a contradiction - or to $T^{2} \times[0,1]$ or a twisted $I$-bundle over a Klein bottle. Hence $\pi_{1}(K)$ is a Klein bottle group or a free abelian group of rank 2 .

\section{Geometrically finite Kleinian groups}

The main results of the section are Proposition 4.2, which I have not seen stated before, and Proposition 4.3, which is routine. The following elementary result, Proposition 4.1, which is needed for the proof of 4.2, seems surprisingly difficult to locate in the literature. 
Proposition 4.1. Let $V \subset \mathbb{C}^{N}$ be an affine algebraic set defined over an algebraically closed subfield $F$ of $\mathbb{C}$. Then $V \cap F^{N}$ is a dense subset of $V$ in the classical (complex) topology.

Proof. According to [Nagata 1993, Theorem 3.10.9], if an algebraic set is defined over the algebraically closed subfield $F$ of $\mathbb{C}$, then its irreducible components are defined over $F$.

I will prove the assertion by induction on the dimension of $V$, which is defined to be the maximum of the dimensions of its irreducible components. If $\operatorname{dim} V=0$ then $V=\left\{P_{1}, \cdots, P_{k}\right\}$ is a finite set. Since each irreducible component $\left\{P_{i}\right\}$ of $V$ is defined over $F$, it follows for example from [Isaacs 1993, Corollary 30.3] that $P_{i} \in F^{N}$, which gives the conclusion in this case.

Now suppose that $V \subset \mathbb{C}^{N}$ is an affine algebraic set defined over $F$ and having dimension $d>0$, and that the assertion is true for affine algebraic subsets of $\mathbb{C}^{N}$ defined over $F$ and having dimension less than $d$. We must show that $V \cap F^{N}$ is dense in the classical (complex) topology of $V$. Since the irreducible components of $V$ are defined over $F$, we may assume without loss of generality that $V$ is irreducible.

Let $U$ be a nonempty subset of $V$ which is open in the classical topology. We must show that $U \cap F^{N} \neq \varnothing$. Let $P_{0}$ be a point of $U$. Since $\operatorname{dim} V>0$, there is an irreducible curve $X$ in $\mathbb{C}^{N}$ with $P \in X \subset V$. Let $X_{0} \subset X$ denote the set of smooth points of $X$. Then $X_{0}$ is nonempty and Zariski-open in $X$, and by [Mumford 1976, Theorem 2.33] it is a dense subset of $X$ in the classical topology. Hence $X_{0} \cap U \neq \varnothing$. Fix a (classically) connected component $W$ of $X_{0} \cap U$. Then $W$ is a complex 1-manifold; in particular it contains more than one point, and hence one of the coordinate functions on $\mathbb{C}^{N}$ is nonconstant on $W$. If $c$ denotes such a coordinate function, then $c \mid W$ is a nonconstant holomorphic function on the connected complex 1-manifold $W$, and is therefore an open map to $\mathbb{C}$. Since $F$ is dense in $\mathbb{C}$, it follows that $c(W) \cap F \neq \varnothing$. Choose a point $\alpha \in c(W) \cap F$. If we set $V^{\prime}=c^{-1}(\alpha)$, it follows that $U^{\prime}:=V^{\prime} \cap U \neq \varnothing$. But $V^{\prime}$ is an algebraic set defined over $F$. It is a proper subset of $V$ since $c \mid W$ is nonconstant, and since $V$ is irreducible it follows that $\operatorname{dim} V^{\prime}<d$. By the induction hypothesis, $V^{\prime} \cap F^{N}$ is dense in the classical topology of $V^{\prime}$, and hence $U^{\prime} \cap F^{N} \neq \varnothing$. In particular $U \cap F^{N} \neq \varnothing$.

Proposition 4.2. Let $\Gamma$ be a nonelementary, finitely generated, torsion-free Kleinian group. Then $\Gamma$ is isomorphic to a Kleinian group $\Gamma_{1}$ such that (i) $\Gamma_{1}$ is geometrically finite, (ii) every maximal parabolic subgroup of $\Gamma_{1}$ has rank 2 , and (iii) $\Gamma_{1} \leq \mathrm{PSL}_{2}(E)$ for some number field $E$.

Proof. If $M$ denotes the orientable hyperbolic 3-manifold $\mathbb{H}^{3} / \Gamma$, we have $\Gamma \cong$ $\pi_{1}(M)$. I claim: 
4.2.1. $\Gamma$ is isomorphic to $\pi_{1}\left(M_{0}\right)$ for some geometrically finite orientable hyperbolic 3-manifold $M_{0}$ having no rank-1 cusps.

Indeed, 4.2.1 is obvious in the case where $M$ is closed, since we may then take $M_{0}=M$. If $M$ is not closed, let us fix a truncation $K$ of $\mathscr{C}_{1}(M)$, and denote by $\mathscr{T}$ the union of all torus components of $\partial K$. According to the main theorem of [Sullivan 1981], $\Gamma$ has only finitely many conjugacy classes of maximal parabolic subgroups; hence by Proposition 3.5, $\mathcal{T}$ has only finitely many components, i.e., it is compact. It then follows from the relative core theorem of [McCullough 1986] that there is a compact, connected submanifold $K_{0} \supset \mathscr{T}$ of $K$ such that the inclusion homomorphism $\pi_{1}\left(K_{0}\right) \rightarrow \pi_{1}(K)$ is an isomorphism. According to [Shalen 2010, Lemma 3.4], there is a compact, irreducible, 3-dimensional submanifold $K_{1}$ of $M$ such that $K_{1} \supset K_{0}$, and such that the inclusion homomorphism $\pi_{1}\left(K_{0}\right) \rightarrow \pi_{1}\left(K_{1}\right)$ is surjective. It follows that $K_{1} \supset \mathscr{T}$ and that the inclusion homomorphism $\pi_{1}\left(K_{1}\right) \rightarrow \pi_{1}(K)$ is an isomorphism. Since $\pi_{1}\left(K_{1}\right) \cong \Gamma$ is torsion-free and nonabelian, and hence infinite, it follows from [Shalen 2010, Proposition 3.9] that $K_{1}$ is aspherical; on the other hand, $K$ is homotopy equivalent to the hyperbolic manifold $M$ and is therefore aspherical. Hence the inclusion $K_{1} \rightarrow K$ is a homotopy equivalence, and so $K_{1}$ is a strong deformation retract of $K$. Since $K$ has no essential tori by Proposition 3.5, any singular essential torus $f: T^{2} \rightarrow K_{1}$ would be homotopic in $K$ to a map $g$ of $T^{2}$ into $\mathscr{T} \subset K_{1}$; since $K_{1}$ is a strong deformation retract of $K$, the maps $f$ and $g$ would be homotopic in $K_{1}$, a contradiction to the essentiality of $f$. Hence $K_{1}$ has no essential tori. We have $\partial K_{1} \neq \varnothing$ since $M$ is noncompact. Since $\pi_{1}(K)$ is isomorphic to the nonelementary Kleinian group $\Gamma$, it has no abelian subgroup of finite index. It therefore follows from Proposition 3.8 that $K_{1}$ is diffeomorphic to $\mathscr{C}_{1}\left(M_{0}\right)$ for some geometrically finite, orientable hyperbolic 3-manifold $M_{0}$ having no rank-1 cusps. In particular we have $\Gamma \cong \pi_{1}(M) \cong \pi_{1}\left(K_{1}\right) \cong \pi_{1}\left(M_{0}\right)$. Thus 4.2.1 is established in all cases.

Let $\operatorname{Hom}\left(\Gamma, \mathrm{SL}_{2}(\mathbb{C})\right)$ denote the set of all representations of $\Gamma$ in $\mathrm{SL}_{2}(\mathbb{C})$. Let $\operatorname{Hom}\left(\Gamma, \mathrm{SL}_{2}(\mathbb{C})\right)^{*}$ denote the subset of $\operatorname{Hom}\left(\Gamma, \mathrm{SL}_{2}(\mathbb{C})\right)$ consisting of all representations $\rho$ such that $\rho$ maps each parabolic element of $\Gamma$ to an element of trace \pm 2 in $\mathrm{SL}_{2}(\mathbb{C})$. Fix a generating set $x_{1}, \ldots, x_{t}$ of $\Gamma$, and define a map of sets

$$
\Phi: \operatorname{Hom}\left(\Gamma, \mathrm{SL}_{2}(\mathbb{C})\right) \rightarrow \mathrm{SL}_{2}(\mathbb{C})^{t} \subset\left(M_{2}\right)^{t}
$$

by $\Phi(\rho)=\left(\rho\left(x_{i}\right)\right)_{1 \leq i \leq t}$. Then

$$
R:=\Phi\left(\operatorname{Hom}\left(\Gamma, \mathrm{SL}_{2}(\mathbb{C})\right)\right) \subset\left(M_{2}\right)^{t}
$$

is readily seen to be an affine algebraic subset of $\left(\mu_{2}\right)^{t}$ (see [Shalen 2002, Subsection 4.1]). Furthermore,

$$
R^{*}:=\Phi\left(\operatorname{Hom}\left(\Gamma, \mathrm{SL}_{2}(\mathbb{C})\right)^{*}\right) \subset R
$$


is also an algebraic set. (In the notation of [Shalen 2002, Subsection 4.4], $R^{*}$ is the locus of zeros within $R$ of the polynomials $I_{\gamma}^{2}-4$, where $\gamma$ ranges over the parabolics in $\Gamma$.)

Now let $P: \mathrm{SL}_{2}(\mathbb{C}) \rightarrow \mathrm{PSL}_{2}(\mathbb{C})$ denote the quotient map. Let $\mathscr{F} \mathscr{G}$ denote the set of all points of $R$ of the form $\Phi(\rho)$, where $\rho: \Gamma \rightarrow \mathrm{SL}_{2}(\mathbb{C})$ is a representation of $\Gamma$ such that $P \circ \rho$ is faithful and $P \circ \rho(\Gamma)$ is a geometrically finite Kleinian group of which all maximal parabolic subgroups have rank 2 . If $M_{0}$ is the hyperbolic manifold given by 4.2.1, then according to [Culler and Shalen 1983, Proposition 3.1.1], the discrete faithful representation of $\pi_{1}\left(M_{0}\right)$ in $\operatorname{PSL}_{2}(\mathbb{C})$ defined by the hyperbolic structure of $M_{0}$ may be lifted to a representation $r: \pi_{1}\left(M_{0}\right) \rightarrow \mathrm{SL}_{2}(\mathbb{C})$; precomposing $r$ with an isomorphism of $\Gamma$ onto $\pi_{1}\left(M_{0}\right)$ gives a representation $\rho$ such that $\Phi(\rho) \in \mathscr{F} \mathscr{G}$. Hence:

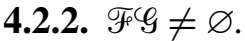

Since a discrete rank-2 free abelian subgroup of $\mathrm{SL}_{2}(\mathbb{C})$ must be parabolic, we have $\mathscr{F F} G \subset R^{*}$. Now I claim:

\subsubsection{The subset $\mathscr{F} \varphi\left(G\right.$ is open in $R^{*}$.}

To prove 4.2 .3 one must show, given a point $\Phi\left(\rho_{0}\right) \in \mathscr{F} \mathscr{G}$, that some neighborhood of $\Phi\left(\rho_{0}\right)$ in $R^{*}$ is contained in $\mathscr{F} \mathscr{G}_{\text {. Here }} P \circ \rho_{0}$ is faithful, $\Gamma_{0}:=P\left(\rho_{0}(\Gamma)\right)$ is discrete and geometrically finite, and all its maximal parabolic subgroups have rank 2. Since the Kleinian group $\Gamma_{0}$ is geometrically finite, it follows from [Matsuzaki and Taniguchi 1998, Theorem 3.7] that there is a finite-sided Dirichlet polyhedron for $\Gamma_{0}$. It then follows from the proof of [Marden 1974, Proposition 9.2] that $\Phi\left(\rho_{0}\right)$ has a neighborhood in $R^{*}$ consisting of points of the form $\Phi(\rho)$, where $\rho$ is a faithful representation such that $P \circ \rho(\Gamma)$ is discrete and geometrically finite, and hence $\Phi(\rho) \in \mathscr{F} \mathscr{G}$. This completes the proof of 4.2.3.

(The definition of a Kleinian group used in [Marden 1974] includes the condition that the group have a nonempty set of discontinuity on the sphere at infinity, although this condition does not appear to be used in the proof of [Marden 1974, Proposition 9.2]. This is why, in the proof of 4.2.3 given above, I have had to quote the proof of [Marden 1974, Proposition 9.2] rather than the statement; the latter result, as stated, does not cover the case in which the set of discontinuity $\Omega_{0} \subset S_{\infty}$ of $\Gamma_{0}$ is empty. An alternative approach in the case $\Omega_{0}=\varnothing$ is to apply [Matsuzaki and Taniguchi 1998, Proposition 3.1] to the manifold $M_{0}=\mathbb{H}^{3} / \Gamma_{0}$ to deduce that $\mathscr{C}_{1}\left(M_{0}\right)$ has empty boundary. It then follows from the definition of a truncation that $\mathscr{b}_{1}\left(M_{0}\right)$ has a truncation whose boundary components are all tori. Hence by Proposition 3.7, $M$ has finite volume. In the finite-volume case, 4.2.3 is a well-known consequence of the results of [Garland 1967].)

Let 9 denote the set of all points of $R^{*}$ whose coordinates are algebraic numbers. Since $9 y$ is the locus of zeros of a set of polynomial equations with integer 
coefficients, we may apply Proposition 4.1, taking $F$ to be the algebraic closure of $\mathbb{Q}$ in $\mathbb{C}$, to deduce that $\mathscr{Y}$ is dense in $R^{*}$. In view of 4.2.2 and 4.2.3 it follows that $\mathscr{Y} \cap \mathscr{F} \mathscr{G} \neq \varnothing$. Let $\rho_{1}$ be a representation such that $\Phi\left(\rho_{1}\right) \in \mathcal{Y} \cap \mathscr{F} \mathscr{G}$, and set $\Gamma_{1}=P\left(\rho_{1}(\Gamma)\right)$ under the quotient homomorphism $\mathrm{SL}_{2}(\mathbb{C}) \rightarrow \mathrm{PSL}_{2}(\mathbb{C})$. Since $\Phi\left(\rho_{1}\right) \in \mathscr{F} \mathscr{G}$, the group $\Gamma_{1}$ is discrete, is isomorphic to $\Gamma$, and satisfies conditions (i) and (ii) of the conclusion of the proposition. Since $\Phi\left(\rho_{1}\right) \in \mathcal{Y}$, there is a number field $E$ containing the coordinates of $\Phi\left(\rho_{1}\right)$. It follows that $\rho_{1}(\Gamma) \leq \mathrm{SL}_{2}(E)$, and condition (iii) of the conclusion follows.

Proposition 4.3. Let $\Gamma$ be a geometrically finite, torsion-free Kleinian group, and let $R$ be a positive real number. Then the set of loxodromic elements of $\Gamma$ having length less than $R$ is a union of finitely many conjugacy classes.

Proof. Set $M:=\mathbb{\boxplus}^{3} / \Gamma$. Fix a truncation $K$ of $\mathscr{C}_{1}(M)$, and let $X_{1}, \ldots, X_{k}$ denote the standard cusp neighborhoods bounded by the torus components of $\partial K$. Since $\Gamma$ is geometrically finite, $K$ is compact (cf. Section 3.2). Let $D$ denote the intrinsic diameter of $K$, so that any two points of $K$ are joined by a path of length at most $D$. Fix a base point $\star \in K$, and fix a point $\widetilde{\star} \in \mathbb{H}^{3}$ that maps to $\star$ under the quotient map $\mathbb{\boxplus}^{3} \rightarrow M$. The point $\widetilde{\star}$ determines an isomorphism $J: \Gamma \rightarrow \pi_{1}(M, \star)$.

Let $\gamma \in \Gamma$ be a loxodromic element of length at most $R$. Then the conjugacy class of $J(\gamma) \in \pi_{1}(M, \star)$ is represented by an oriented closed geodesic $C$ in $M$ with length at most $R$. In the notation of Section 3.2 we have $|C| \subset \mathscr{C}(M) \subset \mathscr{C}_{1}(M)$. Since $\gamma$ is loxodromic, $|C|$ cannot be contained in any of the $X_{i}$. Hence $|C| \cap K \neq \varnothing$. Let us fix a point $y \in S^{1}$ such that $C(y) \in K$. Let $\beta$ be a positively oriented loop in $S^{1}$, based at $y$, which defines a generator of $\pi_{1}\left(S^{1}, y\right)$; then $c:=C \circ \beta$ is a loop in $M$ based at $\beta(y)$. Let $\alpha$ be a path in $K$ which begins at $\star$, ends at $\beta(y)$, and has length at most $D$. Then $c^{\prime}=\alpha \star c \star \bar{\alpha}$ is a loop based at $\star$ which has length at most $R+2 D$, and $\left[c^{\prime}\right]$ is conjugate to $J(\gamma)$ in $\pi_{1}(M, \star)$. Hence $\gamma^{\prime}=J^{-1}\left(\left[c^{\prime}\right]\right)$ is conjugate to $\gamma$ in $\Gamma$, and $d\left(\widetilde{\star}, \gamma^{\prime} \cdot \widetilde{\star}\right) \leq R+2 D$. Since $\Gamma$ is discrete there are only finitely many elements of $\Gamma$ that displace $\widetilde{\star}$ by a distance at most $R+2 D$, and the conclusion follows.

Corollary 4.4. Let $\widetilde{\Gamma}$ be a subgroup of $\mathrm{SL}_{2}(\mathbb{C})$ which maps isomorphically onto a geometrically finite, torsion-free Kleinian group under the quotient homomorphism $\mathrm{SL}_{2}(\mathbb{C}) \rightarrow \mathrm{PSL}_{2}(\mathbb{C})$. Let $\tau \neq \pm 2$ be a complex number. Then the set of elements of $\widetilde{\Gamma}$ having trace $\tau$ is a union of finitely many conjugacy classes.

Proof. Let $\Gamma$ denote the image of $\widetilde{\Gamma}$ on $\operatorname{PSL}_{2}(\mathbb{C})$. If $\gamma \in \widetilde{\Gamma}$ has trace $\tau$, then $[\gamma] \in \Gamma$ is loxodromic since $\tau \neq \pm 2$; and if $l \in(0, \infty)$ and $\theta \in \mathbb{R} / 2 \pi \mathbb{R}$ denote the length and twist angle of $\gamma$, then the quantity $2 \cosh ((l+i \theta) / 2)$, which is well-defined up to sign, is equal to $\pm \tau$. Hence all elements of $\widetilde{\Gamma}$ with trace $\tau$ map to loxodromic elements of the same length in $\Gamma$, and the assertion of the corollary follows from Proposition 4.3. 


\section{Dehn filling}

The main result of this section is Proposition 5.2, which I discussed in the introduction; as I pointed out there, it builds on ideas from [Allman and Hamilton 1999]. Lemma 5.1 will be needed for the proof.

Lemma 5.1. Let $K$ be a compact irreducible orientable 3-manifold with no essential singular tori. Let $T$ be a torus component of $\partial K$, and let $x$ denote a base point in $T$. Suppose that the inclusion homomorphism $\pi_{1}(T, x) \rightarrow \pi_{1}(K, x)$ is injective, and let $P$ denote its image. Then there exist a compact, irreducible orientable 3manifold $K^{\prime}$, a component $T^{\prime}$ of $\partial K^{\prime}$, a base point $x^{\prime} \in T^{\prime}$ and a homomorphism $J: \pi_{1}(K, x) \rightarrow \pi_{1}\left(K^{\prime}, x^{\prime}\right)$, such that

(1) every component of $\partial K^{\prime}$ is a torus;

(2) there are no essential tori in $K^{\prime}$; and

(3) $J \mid P$ is an isomorphism of $P$ onto the image of the inclusion homomorphism $\pi_{1}\left(T^{\prime}, x^{\prime}\right) \rightarrow \pi_{1}\left(K^{\prime}, x^{\prime}\right)$.

Proof. Let $T_{1}, \ldots, T_{k}$ denote the torus components of $\partial K$, indexed so that $T_{1}=$ $T$. If $T_{1}, \ldots, T_{k}$ are the only components of $\partial K$, the conclusion of the lemma follows upon setting $K^{\prime}=K$ and taking $J$ to be the identity map. I will therefore assume that $\partial K$ has one or more boundary component of genus greater than 1 ; let $F_{1}, \ldots, F_{n}$ denote the higher-genus components of $\partial K$, and let $g_{j}>1$ denote the genus of $F_{j}$.

First consider the case in which $K$ is boundary-irreducible. For $j=1, \ldots, n$, the construction of [Fujii 1990] gives a compact orientable hyperbolic 3-manifold $Q_{j}$ with connected totally geodesic boundary such that $\partial Q_{j}$ has genus $g_{j}$. Since $Q_{i}$ has totally geodesic boundary, it follows from [Bonahon 2002, Theorem 2.1] that $Q_{i}$ is irreducible and boundary-irreducible, $Q_{i}$ has no essential singular tori and $\left(Q_{i}, \partial Q_{i}\right)$ has no essential singular annuli (see Section 3.4).

Let $K^{\prime}$ denote the orientable 3-manifold obtained from the disjoint union of $K$ and $Q_{1}, \ldots, Q_{n}$ by gluing $F_{j}$ to $\partial Q_{j}$ via some (arbitrarily chosen) diffeomorphism $\eta_{j}: F_{j} \rightarrow \partial Q_{j}$ for $j=1, \ldots, n$. Since $K$ and each $Q_{i}$ are boundary-irreducible, the surface $F=F_{1} \cup \cdots \cup F_{n}$ is incompressible in $K^{\prime}$. I claim that $K^{\prime}$ is irreducible and boundary-irreducible, and has no essential singular tori.

To prove irreducibility, suppose that $\Sigma \subset K^{\prime}$ is a 2-sphere. After a small isotopy we may assume that $\Sigma$ meets $F$ transversally. I will prove by induction on the number $c$ of components of $\Sigma \cap F$ that $\Sigma$ bounds a ball in $K^{\prime}$. The case $c=0$ follows from the irreducibility of $K$ and the $Q_{j}$. If $c>0$, each component of $\Sigma \cap F$ bounds a disk in $F$ since $F$ is incompressible. Among all disks in $F$ bounded by components of $\Sigma \cap F$, choose one, say $D$, which is minimal with respect to inclusion. Then $C:=\partial D$ bounds two disks $E_{1}, E_{2} \subset \Sigma$, and each $E_{i} \cup D$ is a 
2-sphere $\Sigma_{i}^{\prime}$ which is isotopic by a small isotopy to a 2-sphere meeting $F$ in fewer than $c$ components. By the induction hypothesis, each $\Sigma_{i}^{\prime}$ bounds a ball $B_{i}$. We have either $B_{1} \cap B_{2}=D$ or, after possibly reindexing the $B_{i}$, that $B_{1} \subset B_{2}$. In the first case $B_{1} \cup B_{2}$ is a ball bounded by $\Sigma$, and in the second case $\overline{B_{2}-B_{1}}$ is a ball bounded by $\Sigma$.

The proof of boundary-irreducibility is somewhat similar. Suppose that $\Delta \subset K^{\prime}$ is a properly embedded disk which meets $F$ transversally. I will prove by induction on the number $c$ of components of $\Delta \cap F$ that $\partial \Delta$ bounds a disk in $\partial K^{\prime}$. The case $c=0$ follows from the boundary-irreducibility of $K$ and the $Q_{j}$. If $c>0$, each component of $\Delta \cap F$ bounds a disk in $F$ since $F$ is incompressible. Among all disks in $F$ bounded by components of $\Delta \cap F$, choose one, say $D$, which is minimal with respect to inclusion. Then $C:=\partial D$ bounds a disk $E \subset \Delta$, and $(\Delta-E) \cup D$ is a disk $\Delta^{\prime}$ which is isotopic by a small isotopy to a disk meeting $F$ in fewer than $c$ components. By the induction hypothesis, $\partial \Delta=\partial \Delta^{\prime}$ bounds a disk in $\partial K^{\prime}$.

To show that $K^{\prime}$ has no essential singular tori, suppose that $f: T^{2} \rightarrow K^{\prime}$ induces an injection of fundamental groups. After a small homotopy we may assume that $f$ is transverse to $F$. I will prove by induction on the number $c$ of components of $f^{-1}(F)$ that $f$ is homotopic to a map into $\partial K^{\prime}$. The case $c=0$ follows from the fact that $K$ and the $Q_{j}$ have no essential singular tori. Now suppose that $c>0$ and that some component $\gamma$ of $f^{-1}(F)$ bounds a disk $\Delta \subset T^{2}$. Since $F$ is incompressible, $f \mid \gamma$ is homotopically trivial in $F$. Hence there is a map $f^{\prime}: T^{2} \rightarrow K^{\prime}$ which agrees with $f$ outside $\Delta$ and maps $\Delta$ into $F$. Since $K^{\prime}$ is irreducible we have $\pi_{2}\left(K^{\prime}\right)=0$, and hence $f$ is homotopic to $f^{\prime}$. Clearly $f^{\prime}$ is in turn homotopic by a small homotopy to a map $f_{1}^{\prime}$ such that $\left(f_{1}^{\prime}\right)^{-1}(F)$ has fewer than $c$ components. By the induction hypothesis $f_{1}^{\prime}$ is homotopic to a map into $\partial K^{\prime}$, and hence so is $f$.

Now suppose that $c>0$ and that no component $\gamma$ of $f^{-1}(F)$ bounds a disk $\Delta \subset T^{2}$. Since $c>0$ we have $f^{-1}\left(Q_{1} \cup \cdots Q_{n}\right) \neq \varnothing$. After reindexing the $Q_{i}$ we may assume that $f^{-1}\left(Q_{1}\right) \neq \varnothing$. Choose a component $A$ of $f^{-1}\left(Q_{1}\right)$. Since $\left(Q_{1}, \partial Q_{1}\right)$ has no essential annuli, $f \mid A$ is homotopic in $Q_{1}$ to a map of $A$ into $F_{1}$. Hence $f$ is homotopic to a map $f^{\prime}: T^{2} \rightarrow K^{\prime}$ which agrees with $f$ outside $A$ and maps $A$ into $F$. Clearly $f^{\prime}$ is in turn homotopic by a small homotopy to a map $f_{1}^{\prime}$ such that $\left(f_{1}^{\prime}\right)^{-1}(F)$ has fewer than $c$ components. By the induction hypothesis $f_{1}^{\prime}$ is homotopic to a map into $\partial K^{\prime}$, and hence so is $f$.

Now let $J: \pi_{1}(K, x) \rightarrow \pi_{1}\left(K^{\prime}, x^{\prime}\right)$ be the inclusion homomorphism. I claim the conclusions of the lemma hold with this choice of $J$ if we set $T=T^{\prime}$ and $x^{\prime}=x$. By construction $K^{\prime}$ is compact and orientable and its boundary components are tori. I have shown that $K$ is irreducible and contains no essential singular tori. It remains only to observe that Conclusion (iii) of the lemma holds. If $P^{\prime}$ denotes the image of the inclusion homomorphism $\pi_{1}(T, x) \rightarrow \pi_{1}\left(K^{\prime}, x^{\prime}\right)$, the definitions of $J, P$ and $P^{\prime}$ imply that $J(P)=P^{\prime}$. That $J \mid P: P \rightarrow P^{\prime}$ is an isomorphism is tantamount to 
saying that the inclusion homomorphism $\pi_{1}(T, x) \rightarrow \pi_{1}\left(K^{\prime}, x^{\prime}\right)$ is injective; this follows from the boundary-irreducibility of $K^{\prime}$, which I proved above.

This completes the proof of the lemma when $K$ is boundary-irreducible.

Now consider the case in which $\partial K$ is boundary-reducible. Let $D_{1}, \ldots, D_{m}$ be a maximal system of pairwise disjoint, non-boundary-parallel, pairwise nonparallel, properly embedded disks in $K$. Let $E$ be a regular neighborhood of $D_{1} \cup \cdots \cup D_{m}$ in $K$, and set $K_{0}=\overline{K-E}$.

Since the inclusion homomorphism $\pi_{1}(T) \rightarrow \pi_{1}(K)$ is injective, none of the disks $D_{1}, \ldots, D_{m}$ has its boundary in $T$, and hence $T \subset K_{0}$. Let $K_{1}$ denote the component of $K_{0}$ that contains $T$.

Since $K$ is irreducible and has no essential singular tori, and since $K_{1}$ is a component of a manifold obtained from $K$ by splitting it along a collection of pairwise disjoint properly embedded disks, $K_{1}$ is itself irreducible and has no essential singular tori. Furthermore, $T$ is a component of $\partial K_{1}$ and the inclusion $\pi_{1}(T, x) \rightarrow \pi_{1}\left(K_{1}, x\right)$ is injective; I will denote its image by $P_{1}$. On the other hand, the maximality of the family $D_{1}, \ldots, D_{m}$ implies that $K_{1}$ is boundary-irreducible. It therefore follows from the case of the lemma already proved that there exist a compact, irreducible, orientable 3-manifold $K^{\prime}$, a component $T^{\prime}$ of $\partial K^{\prime}$, a base point $x^{\prime} \in T^{\prime}$ and a homomorphism $J_{1}: \pi_{1}\left(K_{1}, x\right) \rightarrow \pi_{1}\left(K^{\prime}, x^{\prime}\right)$ such that conclusions (i)-(iii) of the lemma hold with $K_{1}, P_{1}$ and $J_{1}$ in place of $K, P$ and $J$.

Since $K_{1}$ is a component of a manifold obtained from $K$ by splitting along a family of disks, the inclusion homomorphism $I: \pi_{1}\left(K_{1}, x\right) \rightarrow \pi_{1}(K, x)$ maps $\pi_{1}\left(K_{1}, x\right)$ isomorphically onto a free factor of $\pi_{1}(K, x)$. In particular $I$ has a left inverse $r$ which is a homomorphism. The definitions of $I, P_{1}$ and $P$ imply that $I\left(P_{1}\right)=P$; since $I$ is injective, it restricts to an isomorphism of $P_{1}$ onto $P$. Hence $r$ maps $P$ isomorphically onto $P_{1}$. Since $J_{1}$ maps $P_{1}$ isomorphically onto $P^{\prime}$, the homomorphism $J:=J_{1} \circ r: \pi_{1}(K, x) \rightarrow \pi_{1}\left(K^{\prime}, x^{\prime}\right)$ maps $P$ isomorphically onto $P^{\prime}$, and the lemma is proved in this case as well.

Proposition 5.2. Let $\Gamma$ be a torsion-free geometrically finite Kleinian group such that every maximal parabolic subgroup of $\Gamma$ has rank 2 . Let $P$ be a maximal parabolic subgroup of $\Gamma$. Then there is a finite set $Y \subset P$ such that for every $\gamma \in P-Y$, and every positive integer $m$, there exist a Kleinian group $\widehat{\Gamma}$ and $a$ homomorphism $H: \Gamma \rightarrow \widehat{\Gamma}$ such that $H(\gamma)$ has order $m$ in $\widehat{\Gamma}$. Furthermore, if $Y$ is such a set, then every positive integer is a finitistic order for every $\gamma \in P-Y$.

Proof. Set $M=\mathbb{H}^{3} / \Gamma$, and fix a truncation $K$ of $\mathscr{C}_{1}(M)$. Let $T_{1} \cup \cdots \cup T_{k}$ denote the torus boundary components of $K$. Since $P$ is a maximal parabolic subgroup of $\Gamma$, it follows from Proposition 3.5 that, after possibly reindexing the $T_{i}$ and choosing a base point $x \in T_{1}$, we have an isomorphic identification of $\Gamma$ with $\pi_{1}(K, x)$ under which $P$ is the image of the inclusion homomorphism $\pi_{1}\left(T_{1}, x\right) \rightarrow \pi_{1}(K, x)$. 
I will begin by proving the first assertion in the case where $\Gamma$ has finite covolume. In this case we have $\mathscr{C}_{1}(M)=M$; and if $K$ is a truncation of $M$, then $\partial K$ is a union of tori $T_{1}, \ldots, T_{k}$ by Proposition 3.7. The proof in this case is an application of Thurston's hyperbolic Dehn filling theorem, which is stated and proved as [Petronio and Porti 2000, Theorem 2.1].

The following notation will be borrowed from [Petronio and Porti 2000]. Fix a basis $\lambda_{i}, \mu_{i}$ of $H_{1}\left(T_{i} ; \mathbb{Z}\right)$ for each $i$. Denote by $C$ the set of all coprime pairs of integers, together with a symbol $\infty$. For $c_{1}, \ldots, c_{k} \in C$ denote by $M_{c_{1} \cdots c_{k}}$ the manifold obtained from $K$ as follows: if $c_{i}=\infty$, glue a half-open collar $T_{i} \times[0, \infty)$ to $T_{i}$; if $c_{i}=\left(p_{i}, q_{i}\right)$, glue a solid torus $J_{i}=D^{2} \times S^{1}$ to $K$ along $T_{i}$, by gluing the meridian $S^{1} \times\{1\}$ to a curve representing the class $p_{i} \lambda_{i}+q_{i} \mu_{i}$. Consider the set

$G=\{\infty\} \cup\left\{g \in \mathbb{R}^{2}: g=r \cdot(p, q)\right.$ for some $r>0$ and relatively prime $\left.p, q \in \mathbb{Z}\right\}$.

For $g=r \cdot(p, q) \in G \backslash\{\infty\}$ define $c(g)=(p, q)$ and $\vartheta(g)=2 \pi / r$. Set $c(\infty)=\infty$. Topologize $G$ as a subset of $\mathbb{R}^{2} \cup\{\infty\}=S^{2}$.

According to [Petronio and Porti 2000, Theorem 2.1] there is a neighborhood $\mathscr{F}_{F}$ of $(\infty, \ldots, \infty)$ in $G^{k}$ such that for $\left(g_{1}, \ldots, g_{k}\right) \in \mathscr{F}$ the manifold $M_{c\left(g_{1}\right) \ldots c\left(g_{k}\right)}$ admits the structure of a complete finite-volume hyperbolic cone manifold in which the cone locus consists of the cores $\{0\} \times S^{1}$ of those $J_{i}$ such that $g_{i} \neq \infty$, and the cone angle at the core of $J_{i}$ is $\vartheta\left(g_{i}\right)$.

The inclusion homomorphism $\pi_{1}\left(T_{1}, x\right) \rightarrow \pi_{1}(K, x)$ may be regarded as an isomorphism of $\pi_{1}\left(T_{1}, x\right)$ onto $P$. Composing the inverse of this isomorphism with the Hurewicz isomorphism from $\pi_{1}\left(T_{1}, x\right)$ to $H_{1}\left(T_{1}, \mathbb{Z}\right)$, one obtains an isomorphism $e: P \rightarrow H_{1}\left(T_{1}, \mathbb{Z}\right)$. The free abelian group $P$ has a basis consisting of the elements $\mathfrak{l}:=e^{-1}\left(\lambda_{1}\right)$ and $\mathfrak{m}:=e^{-1}\left(\mu_{1}\right)$.

Now choose an integer $B>0$ such that for every $c=(x, y) \in G \backslash\{\infty\}$ with $\max (|x|,|y|)>B$ we have $(c, \infty, \ldots, \infty) \in \mathscr{F}$. Let $Y$ denote the finite subset of $P$ consisting of all elements of the form $\mathfrak{l}^{r} \mathrm{~m}^{s}$ as $r$ and $s$ range over all integers of absolute value at most $B$. I will show that the first assertion holds with this choice of $Y$.

Let $\gamma \in P-Y$ be given, and let $m$ be a positive integer. Let us write $\gamma=$ ${ }^{d} \mathfrak{m}^{d q}$, where $p$ and $q$ are relatively prime integers, $d$ is a positive integer, and $\max (|d p|,|d q|)>B$. Hence $\max (m d|p|, m d|q|)>B$. If we set $g=(m d p, m d q)$, it follows that $(g, \infty, \ldots, \infty) \in \mathscr{F}$. By definition we have $c(g)=(p, q)$ and $\vartheta(g)=2 \pi /(m d)$.

The manifold $M_{c(g), \infty, \ldots, \infty}$ is obtained from $K$ by attaching a solid torus $J$ along the boundary component $T_{1}$, and attaching half-open collars to the remaining components of $\partial K$. Here $J$ may be given a product structure $J=D^{2} \times S^{1}$ in such a way that $S^{1} \times\{1\}$ is glued to a simple closed curve representing the class $p \lambda+q \mu \in H_{1}(K ; \mathbb{Z})$. The defining property of $\mathscr{F}$ implies that $M_{c(g), \infty, \ldots, \infty}$ 
admits the structure of a complete finite-volume hyperbolic cone manifold, whose cone locus consists of the core $\{0\} \times S^{1}$ of $J$, and the cone angle at this core is $\vartheta(g)=2 \pi /(m d)$. Since $m d$ is an integer, the cone manifold structure gives $M_{c(g), \infty, \ldots, \infty}$ the structure of a hyperbolic orbifold whose singular locus is the core curve; in the neighborhood of any point of the singular locus, the orbifold is topologically modeled on the quotient of $\mathbb{R}^{3}$ by the finite cyclic group $\mathbb{Z} / m d \mathbb{Z}$, acting through rotations about an axis. It follows that the orbifold fundamental group $\pi_{1}^{\text {orb }}\left(M_{c(g), \infty, \ldots, \infty}\right)$ is isomorphic to a Kleinian group; and that if $H$ denotes the inclusion homomorphism (defined modulo inner automorphisms) from $\pi_{1}(K)$ to $\pi_{1}^{\text {orb }}\left(M_{c(g), \infty, \ldots, \infty}\right)$, then $H$ maps $\gamma_{0}=\mathfrak{l}^{p} \mathfrak{m}^{q}$ onto a element of order of $m d$. Hence $H$ maps $\gamma=\gamma_{0}^{d}$ onto an element of order $m$. This proves the first assertion of the proposition in the finite-covolume case.

I now prove the first assertion in the general case. According to Proposition 3.5, $K$ is irreducible and has no essential singular tori. The inclusion homomorphism $\pi_{1}\left(T_{1}, x\right) \rightarrow \pi_{1}(K, x)$ is injective by Proposition 3.5, and it has image $P$ according to our choice of $T_{1}$. Hence we may apply Lemma 5.1, taking $T=T_{1}$. This gives a compact, irreducible orientable 3-manifold $K^{\prime}$, a component $T^{\prime}$ of $\partial K^{\prime}$, a base point $x^{\prime} \in T^{\prime}$ and a homomorphism $J: \pi_{1}(K, x) \rightarrow \pi_{1}\left(K^{\prime}, x^{\prime}\right)$. Furthermore, every component of $\partial K^{\prime}$ is a torus; there are no essential tori in $K^{\prime}$; and if $P^{\prime}$ denotes the image of the inclusion homomorphism $\pi_{1}\left(T^{\prime}, x^{\prime}\right) \rightarrow \pi_{1}\left(K^{\prime}, x^{\prime}\right)$, then $J$ restricts to an isomorphism of $P$ onto $P^{\prime}$.

I claim:

5.2.1. There is a finite set $Y^{\prime} \subset P^{\prime}$ such that for every $\gamma^{\prime} \in P^{\prime}-Y^{\prime}$, and every positive integer $m$, there exist a Kleinian group $\widehat{\Gamma}$ and a homomorphism

$$
h^{\prime}: \pi_{1}\left(K^{\prime}, x^{\prime}\right) \rightarrow \widehat{\Gamma}
$$

such that $h^{\prime}\left(\gamma^{\prime}\right)$ has order $m$ in $\widehat{\Gamma}$.

To prove this, first note that by Proposition 3.8, we have one of the following possibilities:

(i) $K^{\prime}$ is diffeomorphic to a truncation of $\mathscr{C}_{1}\left(M^{\prime}\right)$ for some geometrically finite orientable hyperbolic 3-manifold $M^{\prime}$ having no rank-1 cusps.

(ii) $\pi_{1}\left(K^{\prime}, x^{\prime}\right)$ is free abelian.

(iii) $\pi_{1}\left(K^{\prime}, x^{\prime}\right)$ is a Klein bottle group.

If (i) holds, then since the boundary components of $K^{\prime}$ are all tori, $M^{\prime}$ has finite volume by Proposition 3.7, and we may write $M^{\prime}=\mathbb{H}^{3} / \Gamma^{\prime}$ for some torsion-free Kleinian group $\Gamma^{\prime}$ of finite covolume. Furthermore, we may identify $\pi_{1}\left(K^{\prime}, x^{\prime}\right)$ isomorphically with $\Gamma^{\prime}$ in such a way that $P^{\prime}$ is a maximal parabolic subgroup. In 
this case, 5.2.1 follows from the finite-covolume case of the first assertion of the lemma, which has already been proved.

If (ii) or (iii) holds, I will set $\Gamma^{\prime}=\pi_{1}\left(K^{\prime}, x^{\prime}\right)$. If (ii) holds, then according to the construction of Section 1.2, for every $\gamma^{\prime} \in \Gamma^{\prime}-\{1\}$ and every positive integer $m$, there is a homomorphism $h^{\prime}$ of $\Gamma^{\prime}$ onto a finite cyclic group $Z$ such that $h^{\prime}\left(\gamma^{\prime}\right)$ has order $m$; as $Z$ is in particular isomorphic to a Kleinian group, 5.2.1 holds in this case with $Y^{\prime}=\{1\}$.

If (iii) holds, we may identify $\Gamma^{\prime}$ isomorphically with $\left\langle x, y: y x y^{-1}=x^{-1}\right\rangle$. Suppose that $\gamma^{\prime} \in \Gamma^{\prime}-\{1\}$ and a positive integer $m$ are given. If $\gamma^{\prime}$ is not a power of $x$, its image $\gamma^{\prime \prime}$ under the quotient homomorphism $\Gamma^{\prime} \rightarrow \Gamma^{\prime} /\langle\langle x\rangle\rangle$ is nontrivial. Since $\Gamma^{\prime} /\langle\langle x\rangle\rangle$ is infinite cyclic, the construction of Section 1.2 gives a homomorphism $h^{\prime \prime}$ of $\Gamma^{\prime} /\langle\langle x\rangle\rangle$ onto a finite cyclic group $Z$ such that $h^{\prime \prime}\left(\gamma^{\prime \prime}\right)$ has order $m$, and again 5.2.1 holds with $Y^{\prime}=\{1\}$. If $\gamma^{\prime}=x^{k}$ for some $k \neq 0$, and if $D$ denotes the finite dihedral group $\left\langle u, v: v^{2}=1, u^{m|k|}=1, v u v^{-1}=u^{-1}\right\rangle$, the homomorphism $h^{\prime}: \Gamma^{\prime} \rightarrow D$ defined by $h^{\prime}(x)=u, h^{\prime}(y)=v$ maps $\gamma^{\prime}$ onto an element of order $m$. As $D$ is isomorphic to a Kleinian group, 5.2.1 holds, in this subcase as well, with $Y^{\prime}=\{1\}$. Thus 5.2.1 is established in all cases.

Let $Y$ be the set given by 5.2.1, and let us set $Y=J^{-1}\left(Y^{\prime}\right)$. Let $\gamma$ be any element of $P-Y$ and set $\gamma^{\prime}=J(\gamma)$. Then $\gamma^{\prime} \in P^{\prime}-Y^{\prime}$. Hence for every positive integer $m$ there exist a Kleinian group $\widehat{\Gamma}$ and a homomorphism $h^{\prime}: \Gamma^{\prime} \rightarrow \widehat{\Gamma}$ such that $h^{\prime}(\gamma)$ has order $m$ in $\widehat{\Gamma}$. Setting $h=h^{\prime} \circ J: \Gamma \rightarrow \widehat{\Gamma}$ we find that $h(\gamma)$ has order $m$ in $\widehat{\Gamma}$. This completes the proof of the first assertion of the proposition.

To prove the second assertion, suppose that $\gamma \in P-Y$ and $m \in \mathbb{N}$ are given. Fix a Kleinian group $\widehat{\Gamma}$ and a homomorphism $H: \Gamma \rightarrow \widehat{\Gamma}$ such that $\hat{\gamma}:=H(\gamma)$ has order $m$ in $\widehat{\Gamma}$. The group $\widehat{\Gamma}$ is a subgroup of $\mathrm{SL}_{2}(\mathbb{C})$, which is in turn isomorphic to a subgroup of $\mathrm{GL}_{2}(\mathbb{C})$ (Indeed, the adjoint action of $\mathrm{SL}_{2}(\mathbb{C})$ on its 3-dimensional Lie algebra factors through a faithful representation of $\mathrm{SL}_{2}(\mathbb{C})$.) In particular $\widehat{\Gamma}$ is a linear group, and is therefore residually finite according to [Malcev 1940]. Since the elements $\hat{\gamma}, \hat{\gamma}^{2}, \ldots, \hat{\gamma}^{m-1}$ of $\widehat{\Gamma}$ are nontrivial, there exist a finite group $G$ and a homomorphism $J: \widehat{\Gamma} \rightarrow G$ such that $J\left(\hat{\gamma}^{i}\right)$ is nontrivial for $i=1, \ldots, m-1$. If $h$ denotes the homomorphism $J \circ H: \Gamma \rightarrow G$, then $h(\gamma)^{i}=J\left(\hat{\gamma}^{i}\right)$ is nontrivial for $i=1, \ldots, m-1$, but $h(\gamma)^{m}$ is trivial since $\hat{\gamma}^{m}$ is trivial. Hence $h(\gamma)$ has order $m$ in $G$. This shows that $m$ is a finitistic order for $\gamma$.

\section{Proof of the main theorem}

This section is devoted to the proof of Theorem 1.4.

As in the statement of the theorem, let $\Gamma$ be a finitely generated, torsion-free Kleinian group, let $m>2$ be an integer, and let $X$ denote the set of all elements of $\Gamma$ for which $m$ is a finitistic order. We must show that $\Gamma-X$ is a union of finitely 
many conjugacy classes. If $\Gamma$ is elementary then it is free abelian by Section 3.1, and by Section 1.2 it follows that $X=\Gamma$. For the rest of the proof I will assume that $\Gamma$ is nonelementary.

The required conclusion depends only on the isomorphism class of $\Gamma$. In view of Proposition 4.2, we may therefore assume without loss of generality that

(1) $\Gamma$ is geometrically finite,

(2) every maximal parabolic subgroup of $\Gamma$ has rank 2, and

(3) $\Gamma \leq \mathrm{PSL}_{2}(K)$ for some number field $K$.

Since $m>1$, the identity element 1 of $\Gamma$ does not belong to $X$. Since $\Gamma$ is torsion-free and discrete, every nontrivial element of $\Gamma$ is loxodromic or parabolic. Hence we may write $\Gamma-X=Z_{\ell} \cup Z_{p} \cup\{1\}$, where $Z_{\ell}$ (resp. $Z_{p}$ ) denotes the set of loxodromic (resp. parabolic) elements of $X$. I shall prove the theorem by showing that each of the sets $Z_{\ell}$ and $Z_{p}$ is a union of finitely many conjugacy classes. Note that each of these sets is obviously invariant under conjugation.

Since $\Gamma \leq \operatorname{PSL}_{2}(K)$ by (3) above, and since $\Gamma$ is finitely generated, there is a finite set $S$ of places of $K$ such that $\Gamma \leq \mathrm{PSL}_{2}\left(\mathcal{O}_{K, S}\right)$. After possibly enlarging $S$ we may assume that it contains the infinite places, i.e., that it is admissible. According to [Culler and Shalen 1983, Proposition 3.1.1], there is a subgroup $\widetilde{\Gamma}$ of $\mathrm{SL}_{2}(\mathbb{C})$ such that the quotient homomorphism $\mathrm{SL}_{2}(\mathbb{C}) \rightarrow \mathrm{PSL}_{2}(\mathbb{C})$ restricts to an isomorphism $Q: \widetilde{\Gamma} \rightarrow \Gamma$. Since $\Gamma \leq \mathrm{PSL}_{2}\left(\mathscr{O}_{K, S}\right)$, we have $\widetilde{\Gamma} \leq \mathrm{SL}_{2}\left(\mathrm{O}_{K, S}\right)$.

We apply Proposition 2.7, defining $S$ as above and using the given value of $m>2$. Since $\widetilde{\Gamma} \leq \mathrm{SL}_{2}\left(\mathrm{O}_{K, S}\right)$, the proposition implies in particular that there is a finite set $W \subset \mathcal{O}_{K, S}$ such that every element $\gamma$ of $\widetilde{\Gamma}$ with trace $\gamma \notin W$ has $m$ as a finitistic order. Hence trace $\left(\widetilde{\Gamma}-Q^{-1}(X)\right) \subset W$.

In particular, if we set $\widetilde{Z}_{\ell}=Q^{-1}\left(Z_{\ell}\right)$, then $\operatorname{trace}\left(\widetilde{Z}_{\ell}\right)$ is contained in $W$ and is therefore finite. On the other hand, since $Z_{\ell}$ consists of loxodromic elements, we have $2,-2 \notin \operatorname{trace}\left(\widetilde{Z}_{\ell}\right)$. Since $\Gamma$ is geometrically finite by (1) above, it follows from Corollary 4.4 that $\widetilde{Z}_{\ell}$ contains only finitely many $\widetilde{\Gamma}$-conjugacy classes of elements with any given trace. Hence $\widetilde{Z}_{\ell}$ contains only finitely many conjugacy classes, and therefore so does $Z_{\ell}$.

Since $\Gamma$ is geometrically finite, a truncation of $M=\mathbb{\boxplus}^{3} / \Gamma$ has only finitely many torus components, and hence by Proposition $3.5, \Gamma$ has only finitely many conjugacy classes of maximal parabolic subgroups. Let $k \geq 0$ denote the number of these conjugacy classes, and let $P_{1}, \ldots, P_{k}$ be subgroups representing them. By (2) above, each of the $P_{i}$ has rank 2. According to Proposition 5.2 (and conditions (1) and (2) above), each $P_{i}$ has a finite subset $Y_{i}$ such that every positive integerand in particular $m-$ is a finitistic order for every $\gamma \in P_{i}-Y_{i}$. Hence each element of $Z_{p}$ is conjugate to an element of $\bigcup_{i=1}^{k} Y_{i}$. This shows that $Z_{p}$ is contained in a union of finitely many conjugacy classes, completing the proof of Theorem 1.4. 


\section{References}

[Allman and Hamilton 1999] E. S. Allman and E. Hamilton, "Abelian subgroups of finitely generated Kleinian groups are separable", Bull. London Math. Soc. 31:2 (1999), 163-172. MR 99m:20118 Zbl 0936.20025

[Bonahon 2002] F. Bonahon, "Geometric structures on 3-manifolds", pp. 93-164 in Handbook of geometric topology, edited by R. J. Daverman and R. B. Sher, North-Holland, Amsterdam, 2002. MR 2003b:57021 Zbl 0997.57032

[Culler and Shalen 1983] M. Culler and P. B. Shalen, "Varieties of group representations and splittings of 3-manifolds", Ann. of Math. (2) 117:1 (1983), 109-146. MR 84k:57005 Zbl 0529.57005

[Fujii 1990] M. Fujii, "Hyperbolic 3-manifolds with totally geodesic boundary", Osaka J. Math. 27:3 (1990), 539-553. MR 91k:57044 Zbl 0727.57010

[Garland 1967] H. Garland, "A rigidity theorem for discrete subgroups", Trans. Amer. Math. Soc. 129 (1967), 1-25. MR 35 \#4953 Zbl 0225.22016

[Hindry and Silverman 2000] M. Hindry and J. H. Silverman, Diophantine geometry: an introduction, Graduate Texts in Math. 201, Springer, New York, 2000. MR 2001e:11058 Zbl 0948.11023

[Isaacs 1993] I. M. Isaacs, Algebra: a graduate course, Brooks/Cole, Pacific Grove, CA, 1993. Reprinted Amer. Math. Soc., Providence, 2009. MR 2472787 Zbl 0805.00001

[Jaco and Shalen 1979] W. H. Jaco and P. B. Shalen, Seifert fibered spaces in 3-manifolds, Mem. Amer. Math. Soc. 220, Amer. Math. Soc., Providence, RI, 1979. MR 81c:57010 Zbl 0415.57005

[Magnus 1969] W. Magnus, "Residually finite groups", Bull. Amer. Math. Soc. 75 (1969), 305-316. MR 39 \#2865 Zbl 0196.04704

[Malcev 1940] A. Malcev, "On isomorphic matrix representations of infinite groups", Rec. Math. [Mat. Sbornik] N.S. 8 (50) (1940), 405-422. MR 2,216d

[Marden 1974] A. Marden, "The geometry of finitely generated kleinian groups", Ann. of Math. (2) 99 (1974), 383-462. MR 50 \#2485 Zbl 0282.30014

[Matsuzaki and Taniguchi 1998] K. Matsuzaki and M. Taniguchi, Hyperbolic manifolds and kleinian groups, Oxford University Press, New York, 1998. MR 99g:30055 Zbl 0892.30035

[McCullough 1986] D. McCullough, "Compact submanifolds of 3-manifolds with boundary", Quart. J. Math. Oxford Ser. (2) 37:147 (1986), 299-307. MR 88d:57012 Zbl 0628.57008

[Morgan 1984] J. W. Morgan, "On Thurston's uniformization theorem for three-dimensional manifolds", pp. 37-125 in The Smith conjecture (New York, 1979), edited by J. W. Morgan and H. Bass, Pure Appl. Math. 112, Academic Press, Orlando, FL, 1984. MR 758464 Zbl 0599.57002

[Mumford 1976] D. Mumford, Algebraic geometry, I: complex projective varieties, Grundlehren der Math. Wissenschaften 221, Springer, Berlin, 1976. MR 56 \#11992

[Nagata 1993] M. Nagata, Theory of commutative fields, Translations of Mathematical Monographs 125, American Mathematical Society, Providence, RI, 1993. MR 94j:12001 Zbl 0826.12001

[Neukirch 1999] J. Neukirch, Algebraic number theory, Grundlehren der Math. Wissenschaften 322, Springer, Berlin, 1999. MR 2000m:11104 Zbl 0956.11021

[Petronio and Porti 2000] C. Petronio and J. Porti, "Negatively oriented ideal triangulations and a proof of Thurston's hyperbolic Dehn filling theorem”, Expo. Math. 18:1 (2000), 1-35. MR 2001c: 57017 Zbl 0977.57011 arXiv 9901045

[Ribenboim 1999] P. Ribenboim, The theory of classical valuations, Springer, New York, 1999. MR 2000d:12007 Zbl 0957.12005 
[Shalen 2002] P. B. Shalen, "Representations of 3-manifold groups", pp. 955-1044 in Handbook of geometric topology, edited by R. J. Daverman and R. B. Sher, North-Holland, Amsterdam, 2002. MR 2003d:57002 Zbl 1012.57003

[Shalen 2010] P. B. Shalen, "Small optimal Margulis numbers force upper volume bounds", preprint, 2010. To appear in Trans. Amer. Math. Soc. arXiv 1010.2736

[Shalen 2011a] P. B. Shalen, "A generic Margulis number for hyperbolic 3-manifolds", pp. 103-109 in Topology and geometry in dimension three, edited by W. Li et al., Contemp. Math. 560, Amer. Math. Soc., Providence, RI, 2011. MR 2866926

[Shalen 2011b] P. B. Shalen, "Trace fields and Margulis numbers”, 2011. arXiv 0902.1011v2

[Sullivan 1981] D. Sullivan, “A finiteness theorem for cusps”, Acta Math. 147:3-4 (1981), 289-299. MR 83f:30043 Zbl 0502.57004

[Waldhausen 1968] F. Waldhausen, "On irreducible 3-manifolds which are sufficiently large", Ann. of Math. (2) 87 (1968), 56-88. MR 36 \#7146 Zbl 0157.30603

Received May 8, 2011. Revised November 15, 2011.

Peter B. Shalen

Department of Mathematics, Statistics, and Computer Science (M/C 249)

UNIVERSITY OF ILLINOIS AT CHICAGO

851 S. MORGAN ST.

CHICAGO IL 60607-7045

UNITED STATES

shalen@math.uic.edu 


\title{
PACIFIC JOURNAL OF MATHEMATICS
}

\author{
http://pacificmath.org \\ Founded in 1951 by \\ E. F. Beckenbach (1906-1982) and F. Wolf (1904-1989)
}

\section{EDITORS}

V. S. Varadarajan (Managing Editor)

Department of Mathematics

University of California

Los Angeles, CA 90095-1555

pacific@math.ucla.edu

Vyjayanthi Chari

Department of Mathematics

University of California

Riverside, CA 92521-0135

chari@math.ucr.edu

\section{Robert Finn}

Department of Mathematics Stanford University

Stanford, CA 94305-2125

finn@math.stanford.edu

Kefeng Liu

Department of Mathematics

University of California

Los Angeles, CA 90095-1555

liu@math.ucla.edu
Darren Long

Department of Mathematics

University of California

Santa Barbara, CA 93106-3080

long@math.ucsb.edu

Jiang-Hua Lu

Department of Mathematics

The University of Hong Kong

Pokfulam Rd., Hong Kong jhlu@maths.hku.hk

Alexander Merkurjev

Department of Mathematics

University of California

Los Angeles, CA 90095-1555

merkurev@math.ucla.edu
Sorin Popa

Department of Mathematics University of California

Los Angeles, CA 90095-1555 popa@math.ucla.edu

Jie Qing

Department of Mathematics

University of California

Santa Cruz, CA 95064

qing@cats.ucsc.edu

Jonathan Rogawski

Department of Mathematics

University of California

Los Angeles, CA 90095-1555

jonr@math.ucla.edu

\section{PRODUCTION}

pacific@math.berkeley.edu

\section{SUPPORTING INSTITUTIONS}

ACADEMIA SINICA, TAIPEI

CALIFORNIA INST. OF TECHNOLOGY INST. DE MATEMÁTICA PURA E APLICADA KEIO UNIVERSITY

MATH. SCIENCES RESEARCH INSTITUTE NEW MEXICO STATE UNIV.

OREGON STATE UNIV.

\author{
STANFORD UNIVERSITY \\ UNIV. OF BRITISH COLUMBIA \\ UNIV. OF CALIFORNIA, BERKELEY \\ UNIV. OF CALIFORNIA, DAVIS \\ UNIV. OF CALIFORNIA, LOS ANGELES \\ UNIV. OF CALIFORNIA, RIVERSIDE \\ UNIV. OF CALIFORNIA, SAN DIEGO \\ UNIV. OF CALIF., SANTA BARBARA
}

\author{
UNIV. OF CALIF., SANTA CRUZ \\ UNIV. OF MONTANA \\ UNIV. OF OREGON \\ UNIV. OF SOUTHERN CALIFORNIA \\ UNIV. OF UTAH \\ UNIV. OF WASHINGTON \\ WASHINGTON STATE UNIVERSITY
}

These supporting institutions contribute to the cost of publication of this Journal, but they are not owners or publishers and have no responsibility for its contents or policies.

See inside back cover or pacificmath.org for submission instructions.

The subscription price for 2012 is US \$420/year for the electronic version, and \$485/year for print and electronic.

Subscriptions, requests for back issues from the last three years and changes of subscribers address should be sent to Pacific Journal of Mathematics, P.O. Box 4163, Berkeley, CA 94704-0163, U.S.A. Prior back issues are obtainable from Periodicals Service Company, 11 Main Street, Germantown, NY 12526-5635. The Pacific Journal of Mathematics is indexed by Mathematical Reviews, Zentralblatt MATH, PASCAL CNRS Index, Referativnyi Zhurnal, Current Mathematical Publications and the Science Citation Index.

The Pacific Journal of Mathematics (ISSN 0030-8730) at the University of California, c/o Department of Mathematics, 969 Evans Hall, Berkeley, CA 94720-3840, is published monthly except July and August. Periodical rate postage paid at Berkeley, CA 94704, and additional mailing offices. POSTMASTER: send address changes to Pacific Journal of Mathematics, P.O. Box 4163, Berkeley, CA 94704-0163.

PJM peer review and production are managed by EditFLOW ${ }^{\mathrm{TM}}$ from Mathematical Sciences Publishers.

PUBLISHED BY PACIFIC JOURNAL OF MATHEMATICS

at the University of California, Berkeley 94720-3840

A NON-PROFIT CORPORATION

Typeset in LATEX

Copyright $(02012$ by Pacific Journal of Mathematics 


\section{PACIFIC JOURNAL OF MATHEMATICS}

Volume $256 \quad$ No. $1 \quad$ March 2012

On slim double Lie groupoids

NiCOlas ANDruskiewitsch, JEsus OCHOA ARANGO and AlEJANDRO

TIRABOSCHI

Topological classification of quasitoric manifolds with second Betti number 2

19

Suyoung Choi, SeOnjeOng PARK and Dong Youp SuH

Refined Kato inequalities for harmonic fields on Kähler manifolds

DANiEL Cibotaru and PENG ZHU

Deformation retracts to the fat diagonal and applications to the existence of peak solutions of nonlinear elliptic equations

E. Norman Dancer, Jonathan Hillman and Angela Pistoia

Descent for differential Galois theory of difference equations: confluence and $q$-dependence

LuCia Di Vizio and Charlotte HaRdouin

Modulation and natural valued quiver of an algebra

FANG LI

Willmore hypersurfaces with two distinct principal curvatures in $\mathbb{R}^{n+1}$

TONGZHU LI

Variational inequality for conditional pressure on a Borel subset

YuAN Li, ERCAI CHEN and WEN-ChIAO CHENG

New homotopy 4-spheres

DANIEL NASH

Combinatorial constructions of three-dimensional small covers

YASUZO NISHIMURA

On a theorem of Paul Yang on negatively pinched bisectional curvature

AERYEONG SEO

Orders of elements in finite quotients of Kleinian groups

PETER B. Shalen

A new algorithm for finding an 1.c.r. set in certain two-sided cells

JIAN-YI SHI

Addendum to the article Superconnections and parallel transport

FLORIN DUMITRESCU 